INRA Prod. Anim., 2008, 21 (4), 367-386

\title{
Evaluation globale des systèmes de production porcine et leur optimisation au niveau de l'exploitation
}

\author{
M. BONNEAU ${ }^{1}$, J.-Y. DOURMAD ${ }^{1}$, B. LEBRET ${ }^{1}$, M.-C. MEUNIER-SALAÜN ${ }^{1}$, S. ESPAGNOL ${ }^{2}$, \\ Y. SALA ̈̈N $N^{2}$, P. LETERME ${ }^{3}, H$. VAN DER WERF ${ }^{3}$
}

IINRA, UMR 1079 Systèmes d'Elevage, Nutrition Animale et Humaine, F-35590 Saint-Gilles, France

2IFIP Institut du Porc, F-35651 Le Rheu, France

IINRA, UMR 1069 Sol, Agro-hydrosystèmes, Spatialisation, F-35042 Rennes, France

Courriel : Michel.Bonneau@rennes.inra.fr

La durabilité d'un système de production se mesure classiquement selon les trois dimensions de l'environnement, du social et de l'économique. La plupart des travaux conduits dans le cadre du programme «Porcherie verte» correspondaient à des approches monodimensionnelles, voire monodisciplinaires. Il s'agissait par exemple de contribuer à la maîtrise des rejets de phosphore via un meilleur contrôle de l'alimentation des animaux ou à une meilleure connaissance des rejets de gaz à effet de serre selon le procédé utilisé pour le compostage des effluents. Ces approches ont l'avantage de bien se prêter aux études expérimentales classiques. Elles présentent cependant des limites majeures, liées au fait qu'elles ne prennent en compte qu'une facette du problème :

- la conduite d'un atelier d'élevage porcin est soumise à un ensemble varié de contraintes dont l'impact environnemental n'est que l'une des composantes. Il est indispensable de prendre aussi en compte les dimensions sociales (bien-être animal, quantité et qualité du travail), et économiques (coûts de production qui dépendent des performances des animaux et de la valorisation des produits en lien avec leurs qualités) ;

- le chef d'exploitation est l'un des maillons décisionnels fondamentaux en matière de production agricole. C'est lui en effet qui intègre les contraintes multiples qui régissent l'atelier de production porcine en interaction avec les autres ateliers de son exploitation, en particulier avec les productions végétales. Les ateliers de production animale et végétale sont fortement interdépendants au niveau de l'épandage des effluents animaux sur les surfaces, de la fourniture de matières premières alimentaires et de litières pour les animaux et de la compétition pour les ressources en travail et en capital ;

- en restant dans le seul domaine de l'impact environnemental, l'optimisation d'un mode de production sur un seul critère peut conduire à des transferts de pollution d'un compartiment à un autre. On court en effet le risque de créer un problème en voulant en résoudre un autre. C'est ainsi, par exemple, que le traitement des effluents porcins (par compostage ou par traitement biologique) permet certes de résorber des excédents d'azote et donc de limiter l'impact local sur la qualité des eaux, mais peut conduire à aggraver l'impact climatique (rejet direct d'ammoniac et de gaz à effet de serre, utilisation d'énergie pour le traitement, destruction de la valeur fertilisante azotée à remplacer par des engrais minéraux dont la synthèse et le transport sont très «énergivores»...) ;

- toujours dans le domaine de l'impact environnemental, il est fondamental de se situer à différentes échelles. Alors que les impacts sur les sols, la biodiversité ou l'eutrophisation peuvent se raisonner à une échelle assez locale (parcelle, exploitation, bassin versant, région), les impacts sur l'utilisation des terres et le climat doivent être raisonnés à une échelle beaucoup plus vaste, en général mondiale. L'optimisation environnementale à l'échelle de l'exploitation ou, pire encore, à la seule échelle de l'atelier porcin, peut ainsi conduire, comme évoqué plus haut, à des transferts de pollution et à la création de nouveaux problèmes qui peuvent être plus graves que ceux que l'on cherche à résoudre.
Dans la suite de cet article, nous exposerons les résultats obtenus au sein de trois actions de recherche à fort caractère intégratif, qui visaient à surmonter les limites évoquées ci-dessus. La première s'est attachée à une évaluation multicritères de deux modes contrastés de production porcine. La seconde a jeté les premières bases de la construction d'un modèle visant à optimiser le fonctionnement global d'une exploitation agricole comprenant un atelier porc et des productions végétales. La troisième visait à mettre au point une méthode d'évaluation globale de l'impact environnemental prenant en compte, au-delà des impacts directs des facteurs de production mis en œuvre sur l'atelier ou l'exploitation, l'ensemble de leurs impacts indirects qui se font sentir en dehors des limites de l'atelier ou de l'exploitation.

\section{1 / Comparaison multicritè- res de deux types de condui- te d'élevage}

Ce travail (Lebret et al 2004, 2005, 2006a et b, Meunier-Salaün et al 2006, Dourmad et al 2008) se proposait de comparer, à l'échelle de l'atelier de production porcine, deux modes de conduite contrastés en utilisant une batterie de critères relatifs aux performances techniques et économiques, y compris la qualité des produits, au bien-être animal et aux impacts environnementaux. Les caractéristiques des modes de conduite «conventionnel» et «alternatif» sont exposées au tableau 1. Le mode conventionnel correspond à celui utilisé de façon dominante dans le monde entier pour une production 
Tableau 1. Caractéristiques des modes de production comparés au paragraphe 1 (d'après Lebret et al 2004).

\begin{tabular}{c|c}
\hline Conventionnel (caillebotis) & Alternatif (litière + courette) \\
\hline $\begin{array}{c}\text { Claustration totale } \\
\text { Lisier accumulé dans fosse profonde } \\
\text { sous caillebotis total }\end{array}$ & $\begin{array}{c}\text { Accès permanent à courette } \\
\text { extérieure bétonnée } \\
\text { Litière de sciure profonde }\end{array}$ \\
Température constante à $22^{\circ} \mathrm{C}$ & $\begin{array}{c}\text { Température ambiante variable } \\
0,65 \mathrm{~m}^{2} \text { par porc }\end{array}$ \\
$\begin{array}{c}1,3 \mathrm{~m}^{2} \text { par porc à l'intérieur }+1,1 \mathrm{~m}^{2} \\
\text { par porc sur la courette extérieure }\end{array}$ \\
\hline \multicolumn{2}{c}{ Toutes les autres caractéristiques, en particulier } \\
\hline
\end{tabular}

intensive à faible coût. Le logement des animaux à forte densité sur caillebotis intégral avec stockage du lisier dans des fosses situées sous les animaux correspond à la recherche d'un coût d'investissement minimal et d'une réduction de la quantité de travail liée à la gestion des effluents. Le mode alternatif dans lequel des animaux sont logés sur litière et ont accès à un parcours extérieur est généralement perçu comme favorable au bien-être des animaux et ayant un moindre impact sur l'environnement, en particulier en ce qui concerne les odeurs.

Figure 1. Aspects du comportement de porcs en croissance élevés selon deux modes de conduite contrastés, sur caillebotis intégral ou sur litière avec accès à une courette extérieure (d'après Lebret et al 2004).

\section{a. Répartition du budget temps.}

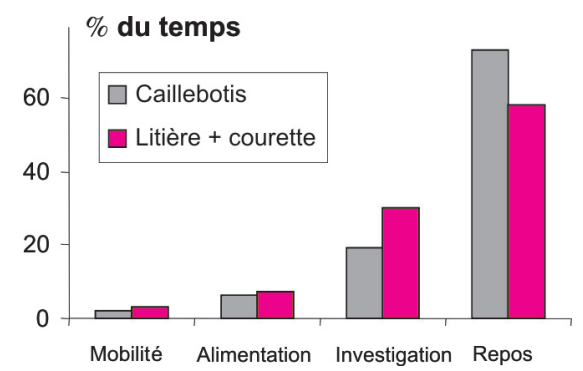

b. Répartition des activités d'investigation.

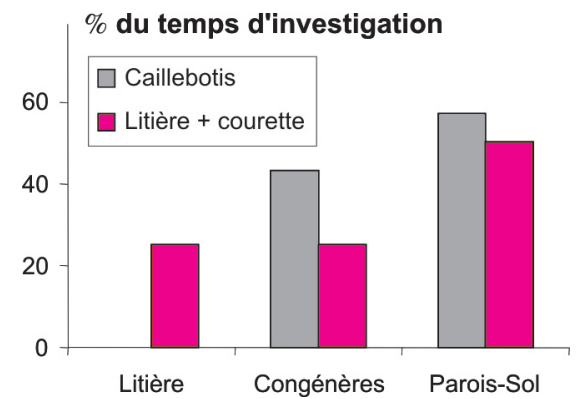

1.1 / Les animaux sur litière avec accès à une courette bénéficient d'un meilleur bien-être que ceux élevés de façon conventionnelle

Comparativement aux animaux élevés sur caillebotis dans un espace plus réduit et sans substrat meuble, les animaux élevés sur litière avec accès à une courette répartissent différemment leurs activités diurnes (figure 1). Ils présentent une activité exploratoire plus importante (figure 1a) dont une partie est tournée vers le substrat dispoêtre considéré comme participant au bien-être des animaux car le fouissage est une activité naturelle chez cette espèce. Ils passent en contrepartie moins de temps à l'activité d'investigation sur les congénères (figure $1 \mathrm{~b}$ ) que les animaux élevés sur caillebotis, activité à risque dans l'apparition de cannibalisme. Par ailleurs, ils ont une motivation moindre pour un objet nouveau

Figure 2. Motivation d'investigation pour un objet nouveau introduit auprès de porcs en croissance élevés selon deux modes de conduite contrastés, sur caillebotis intégral ou sur litière avec accès à une courette extérieure. La motivation est exprimée par la moyenne des durées individuelles de contact des animaux avec l'objet (d'après Meunier-Salaün et al 2006).

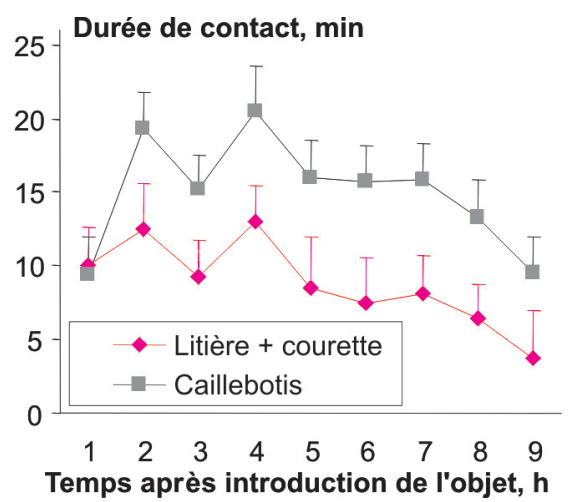
nible, la litière (figure $1 \mathrm{~b}$ ). Ceci peut
Figure 3. Indicateurs de l'état de santé de porcs en croissance élevés selon deux modes de conduite contrastés, sur caillebotis intégral ou sur litière avec accès à une courette extérieure. Pour chaque paramètre la figure donne la fréquence (Fréq.) et l'intensité (Int.) (d'après Lebret et al 2004).

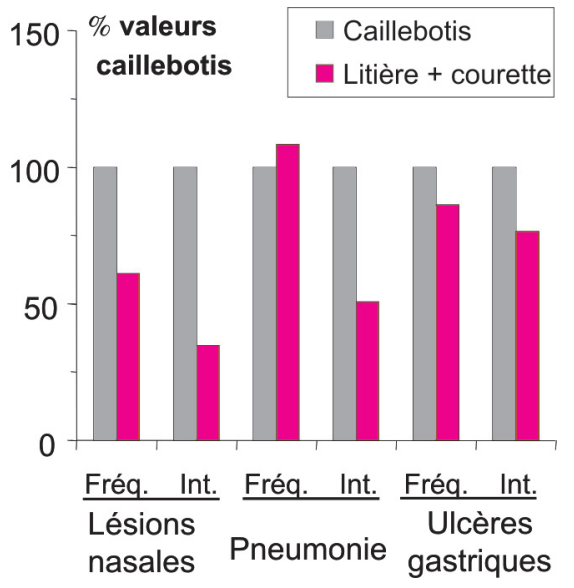

introduit dans leur loge (figure 2), ce qui traduit que leur besoin d'investigation est mieux satisfait dans ces conditions d'élevage. Enfin, l'état de santé des porcs à l'abattage apparaît amélioré (figure 3). Ils sont en particulier moins sensibles aux pathologies des voies respiratoires comme le montrent les moindres fréquence et intensité des lésions nasales et la plus faible intensité des lésions pulmonaires. L'ensemble de ces résultats suggère une amélioration du bien-être des animaux dans le système litière/courette, comparativement au conventionnel.

\section{2 / Les animaux sur litière avec accès à une courette ont de meilleures performances de croissance mais leurs carcasses peuvent être plus grasses}

Les animaux élevés sur litière avec accès à une courette ont consommé davantage d'aliment et ont eu un gain de poids plus rapide, avec la même efficacité alimentaire (figure 4a). Ces meilleures performances sont vraisemblablement liées à la plus faible densité en élevage et à leur meilleur état de santé. Par ailleurs, la température ambiante plus faible a stimulé le niveau d'ingestion spontané des animaux. Ceci indique que le système conventionnel n'est pas optimal pour les performances de croissance des porcs.

La teneur en viande maigre des carcasses peut être réduite $(-2,0$ points 
Figure 4. Performances de croissance et qualité des produits pour des porcs élevés selon deux modes de conduite contrastés, sur caillebotis intégral ou sur litière avec accès à une courette extérieure (d'après Lebret et al 2004 ; les données sont exprimées en pourcentage des valeurs observées pour le groupe conduit sur caillebotis). a. Consommation d'aliment, vitesse de croissance (GMQ) et indice de consommation (IC).

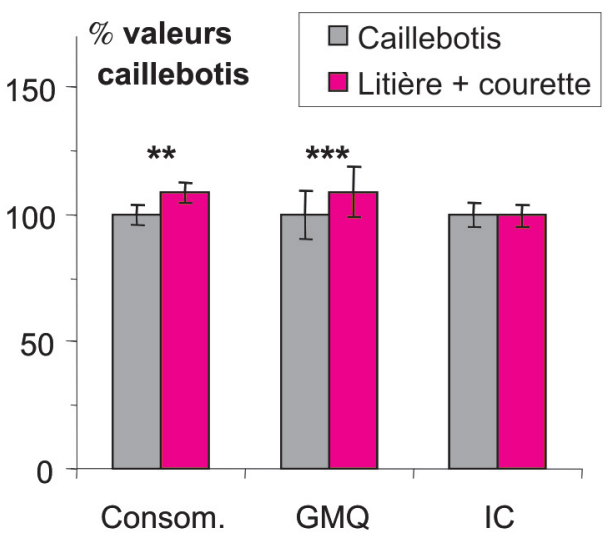

dans la première répétition de l'expérience, figure 4b), mais ceci n'est pas systématique car cette différence n'a pas été observée dans la deuxième répétition. Le mode d'élevage alternatif semble donc accrô̂tre la variabilité de qualité des carcasses. b. Teneur en maigre des carcasses, pertes en eau du muscle après abattage et de la longe.

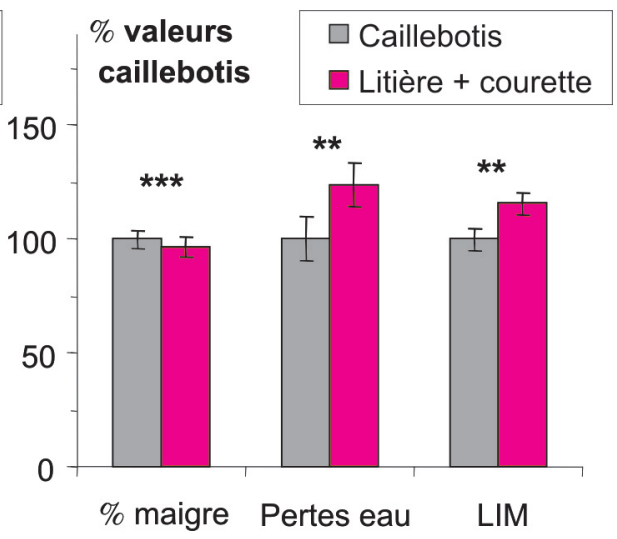

1.3 / Les viandes des animaux sur litière avec accès à une courette peuvent avoir une moindre qualité technologique, mais une meilleure qualité sensorielle

Les critères de qualité technologique (en particulier le $\mathrm{pH}$ ultime des viandes teneur en lipides intramusculaires (LIM)

Figure 5. Caractéristiques sensorielles de la viande de porcs élevés selon deux modes de conduite contrastés, sur caillebotis intégral ou sur litière avec accès à une courette extérieure (d'après Lebret et al 2006b ; $G=$ Gras, $M=$ Maigre).

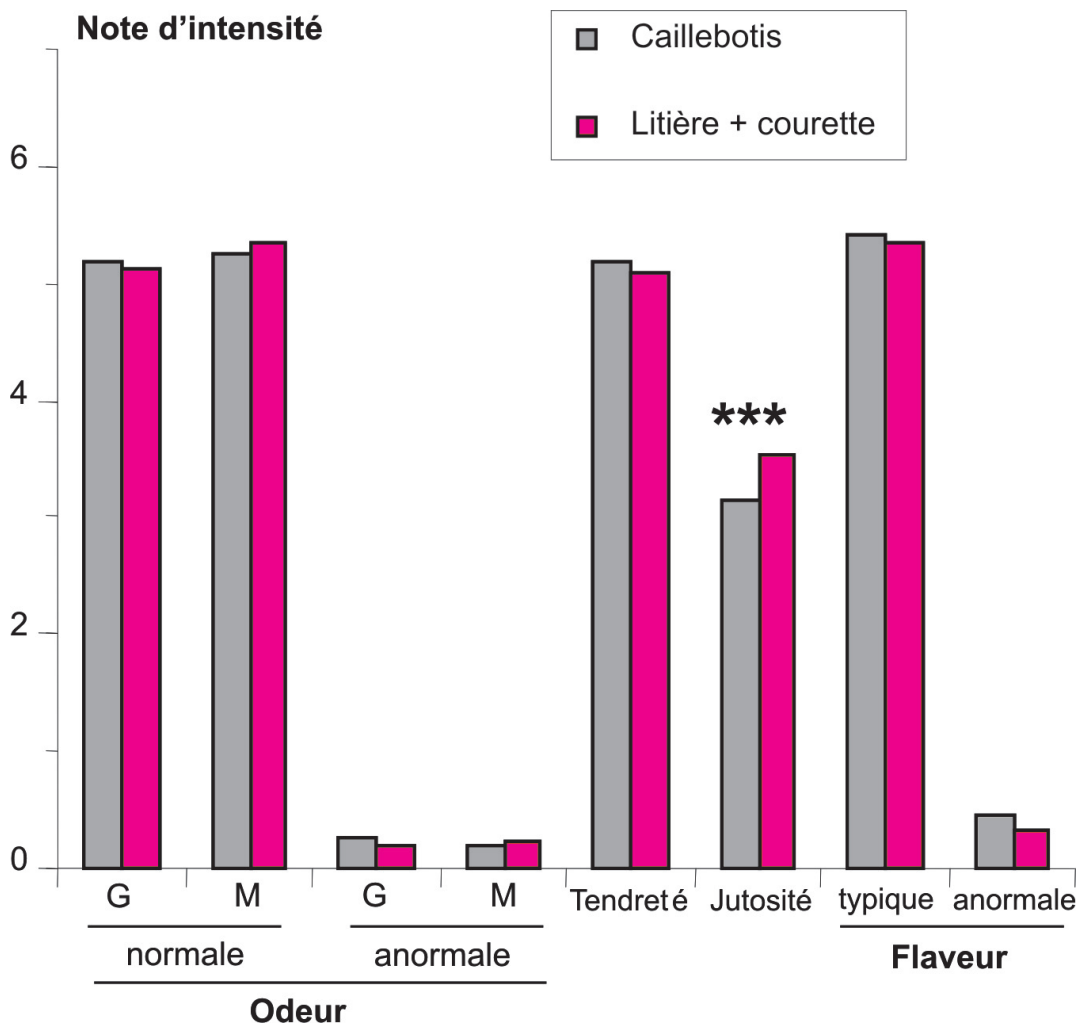

et les pertes en eau) peuvent être négativement affectés dans les muscles du jambon des animaux élevés sur litière avec accès à une courette (figure 4b) ; toutefois ceci n'est pas systématique. L'effet est moins marqué pour les muscles de la longe.

La qualité sensorielle de la viande (notamment la jutosité) évaluée par un jury d'experts est améliorée (figure 5), en relation avec l'accroissement du taux de lipides intramusculaires (figure 4b). Les pertes en eau plus élevées des viandes issues du système litière/courette ne semblent pas influencer la qualité sensorielle.

\section{4 / L'élevage des porcs sur litière avec accès à une cou- rette produit nettement moins d'odeurs désagréables que l'éle- vage conventionnel}

Les flux d'éléments potentiellement à risque pour l'environnement générés dans le système litière-courette sont intermédiaires entre les systèmes «litière» et «lisier». Rappelons que l'élevage sur litière permet d'éliminer des effluents une partie importante de l'azote excrété par les animaux (cf. article sur le devenir des éléments à risque). Cet abattement d'azote peut représenter un avantage en zone d'excédent structurel, mais correspond aussi à une perte de valeur fertilisante azotée dont la production est coûteuse et «énergivore». Cette perte de valeur fertilisante est aussi observée en élevage conventionnel lorsque les effluents sont soumis à un traitement biologique (cf. article sur le devenir des éléments à risque). Une partie de l'azote abattu est émise sous

Figure 6. Concentrations de poussières et émissions d'ammoniac et d'odeurs liés à l'élevage de porcs en croissance selon deux modes de conduite contrastés, sur caillebotis intégral ou sur litière avec accès à une courette extérieure (d'après Lebret et al 2004).

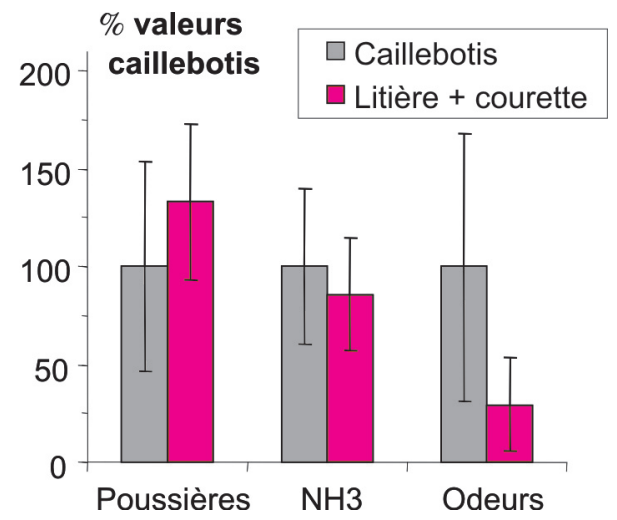


Figure 7. Surcoûts liés au mode de production «litière + courette» (en \% des coûts liés au mode de production conventionnel). Les surcoûts de production sont ventilés entre plusieurs postes (rouge à gris foncé). Les surcoûts pour le consommateur sont calculés sous deux hypothèses selon le pourcentage de la production labélisé (d'après Lebret et al 2005).

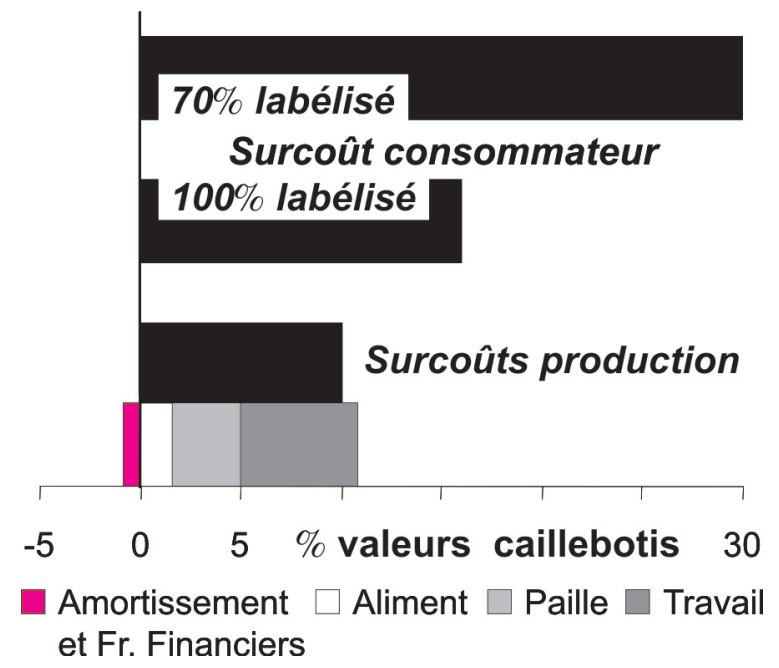

forme de $\mathrm{N}_{2} 0$, gaz qui a un puissant effet de serre (cf. article sur les émissions gazeuses). En contrepartie l'émission de méthane, qui requiert des conditions anaérobies, est réduite. Concernant la qualité de l'air dans les bâtiments (figure 6), le niveau de volatilisation de l'ammoniac et la concentration en poussières sont peu influencés par le système d'élevage, par contre la production d'odeurs est fortement réduite dans le système litière-courette (cf. article sur les nuisances olfactives).

\section{5 / L'élevage des porcs sur} litière avec accès à une courette est plus coûteux, mais il peut être mieux valorisé par un label

Les coûts de production liés à l'élevage «litière + courette» sont environ $10 \%$ plus élevés que ceux de l'élevage conventionnel (Lebret et al 2005, figure 7) : le plus faible coût des amortissements et des frais financiers ne compense pas en effet le surcroît de travail, la plus forte consommation d'aliment et le coût de la paille constituant la litière. Ces coûts de production plus élevés peuvent cependant s'équilibrer par une meilleure valorisation financière des carcasses qui se traduisent, in fine, par un surcoût de 16 à $30 \%$ pour les consommateurs, selon que la totalité ou seulement $70 \%$ de la production peut être valorisée sous label de qualité. Dans sa fourchette basse, ce surcoût est en cohérence avec l'estimation des consentements à payer pour une amélioration globale de la viande de porc et de son mode de production (qualités sanitaire et sensorielle du produit, bienêtre des animaux, impact environnemental) effectuée par Carpentier et al (2003).
Les aspects positifs et négatifs des deux systèmes sont récapitulés au tableau 2. L'avantage du système «litière + courette» pour le bien-être animal, comme ses inconvénients en termes de coût de production, semblent bien établis. Les différences entres les deux systèmes sont faibles pour la qualité des produits. Hormis l'aspect «odeurs», qui est à l'avantage du système alternatif, les différences entre les deux modes de production concernant l'impact environnemental peuvent être très variables en fonction du mode de gestion des effluents.

Cette étude présente l'avantage d'avoir tenté de prendre en compte les différentes dimensions de la durabilité de la production, en y intégrant des évaluations environnementales, sociales (bien-être animal) et économiques (coûts de production et qualité des produits déterminant les possibilités de valorisation). Elle a cependant l'inconvénient de se restreindre aux dimensions de l'atelier de production porcine. Par ailleurs, les résultats obtenus ne sont valides que pour les deux situations étudiées et ne peuvent pas être généralisés facilement. L'étude suivante, exposée au chapitre 2, élargit l'évaluation à l'ensemble d'une exploitation agricole et s'efforce d'être beaucoup plus générique en utilisant la modélisation.

\section{2 / Optimisation au niveau de l'exploitation}

Ce travail (Baudon et al 2005, Dourmad et al 2005, 2006) visait à jeter les premières bases d'un modèle permettant d'optimiser le fonctionnement d'une exploitation agricole avec des ateliers d'élevage.

Tableau 2. Récapitulatif des avantages et inconvénients du mode d'élevage alternatif comparativement au mode conventionnel (+ : à l'avantage du système alternatif ; - à l'avantage du système conventionnel ; d'après Lebret et al 2004, 2005).

\begin{tabular}{|c|c|c|c|}
\hline \multicolumn{2}{|c|}{ Dimensions d'intérêt } & \multicolumn{2}{|r|}{ Alternatif par rapport au conventionnel } \\
\hline \multirow[b]{2}{*}{ Pour la société } & $\begin{array}{l}\text { Bien-être } \\
\text { animal }\end{array}$ & + & $\begin{array}{l}\text { Meilleure expression du répertoire comportemental de l'espèce, } \\
\text { meilleur état de santé }\end{array}$ \\
\hline & $\begin{array}{l}\text { Impact } \\
\text { environnemental }\end{array}$ & $+/-$ & $\begin{array}{l}\text { Moins d'odeurs ; ammoniac et poussières équivalentes ; émission } \\
\text { accrue de } \mathrm{N}_{2} \mathrm{O} \text { et réduite de } \mathrm{CH}_{4} \text { entrainant une émission supérieure } \\
\text { de gaz à effet de serre exprimée en équivalent } \mathrm{CO}_{2} \text {. }\end{array}$ \\
\hline \multirow[b]{2}{*}{$\begin{array}{l}\text { Pour les } \\
\text { producteurs }\end{array}$} & $\begin{array}{l}\text { Coût de } \\
\text { production }\end{array}$ & - & 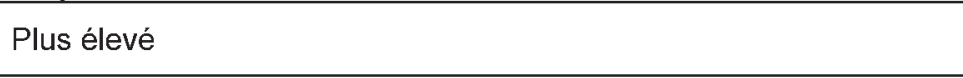 \\
\hline & $\begin{array}{l}\text { Valorisation des } \\
\text { produits pour } \\
\text { le producteur }\end{array}$ & + & Meilleure valorisation des produits au travers de labels de qualité \\
\hline \multirow{2}{*}{$\begin{array}{l}\text { Pour les } \\
\text { consommateurs }\end{array}$} & Prix d'achat & - & Produits labellisés plus chers \\
\hline & $\begin{array}{l}\text { Qualité } \\
\text { des produits }\end{array}$ & $+/-$ & $\begin{array}{l}\text { Différences peu marquées, qualité technologique peut être moindre } \\
\text { et plus variable, qualité sensorielle potentiellement meilleure }\end{array}$ \\
\hline
\end{tabular}


Figure 8. Modélisation des niveaux de rejets en fonction des performances des animaux (haut), de la teneur en protéines des aliments (milieu) ou de la température ambiante dans la salle d'élevage (bas) (d'après Dourmad et al 2002).
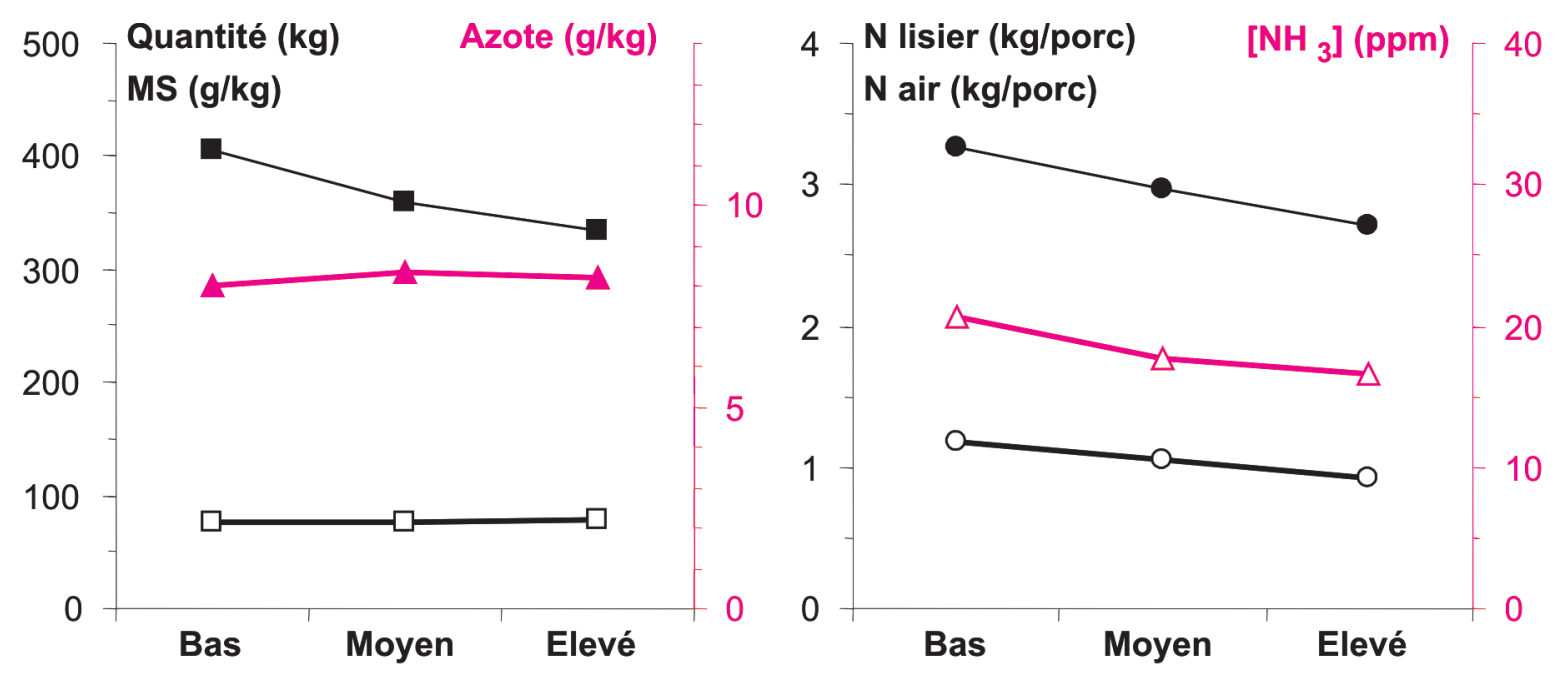

Niveau de performances des animaux (vitesse de croissance et efficacité alimentaire)
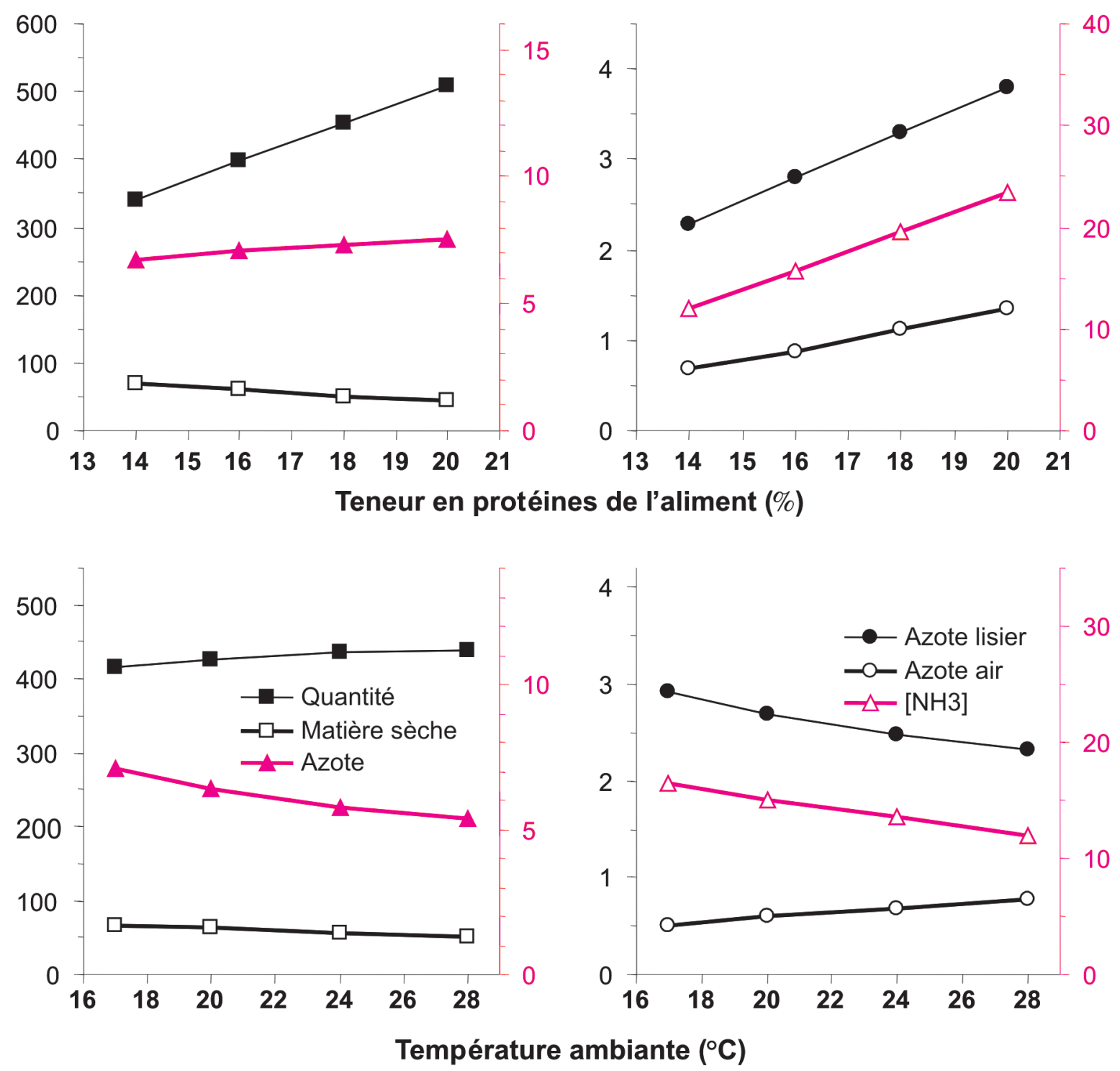
Figure 9. Représentation des compartiments et des flux de matières (azote et phosphore) entre compartiments $(\rightarrow)$ ) dans une exploitation avec élevage porcin. Les éléments en rouge figurent les flux d'entrée $(\rightarrow)$ ) et de sortie $(\rightarrow$; : émissions gazeuses) (d'après Baudon et al 2005).

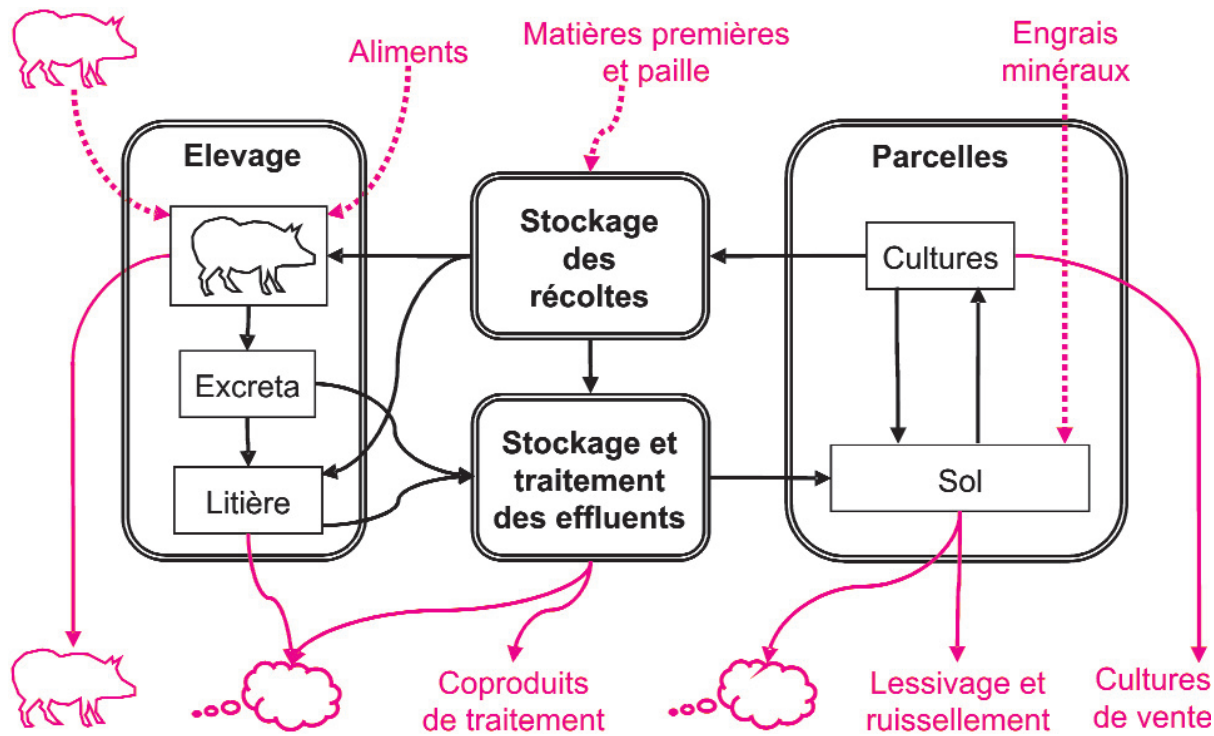

Un premier travail des auteurs (Dourmad et al 2002) avait conduit à un modèle «Elevage» décrivant les flux d'éléments à risque pour l'environnement dans un élevage porcin. Il permettait d'évaluer les rejets potentiellement polluants en fonction des performances des animaux et des conditions réelles de l'élevage, de manière plus précise donc que les approches basées sur des valeurs moyennes standard (figure 8). Mais il restait limité à la production d'effluents par les animaux et ne prenait pas en compte les flux générés par le stockage, le traitement et l'utilisation agronomique des effluents.

Le modèle d'optimisation du fonctionnement d'une exploitation construit ici, a l'ambition de décrire le fonction- nement d'une exploitation comportant un atelier d'engraissement porcin, et en particulier les flux d'éléments en son sein. Il s'agit d'un modèle comportant plusieurs compartiments (figure 9) et décrivant les flux entre compartiments et les échanges avec l'extérieur de l'exploitation.

Cette première ébauche de modèle considère une exploitation de 50 ha de SAU (Surface Agricole Utile) comprenant un élevage porcin qui se limite à un atelier d'engraissement. Un nombre limité de cultures (maïs, blé, colza et pois) est autorisé sur la surface consacrée aux productions végétales. Le module «Elevage» s'appuie sur le modèle «Elevage» évoqué au paragraphe précédent. Les quantités d'élé-

Figure 10. Capacité maximale de production de porcs pour différentes filières de gestion des effluents, sous deux hypothèses de contraintes règlementaires environnementales ( limite 170 UN/ha SPE ; - limites 170 UN/ha SPE et $100 \mathrm{UP}_{2} \mathrm{O}_{5} /$ ha SAU). Dans le cas des filières avec exportation, les triangles pointant vers le haut indiquent que la capacité maximale de production par ha n'a pas de limite calculable et peut dépasser les 210 porcs par ha indiqués sur la figure. La marge brute est calculée pour la production maximale autorisée par la filière (d'après Baudon et al 2005).

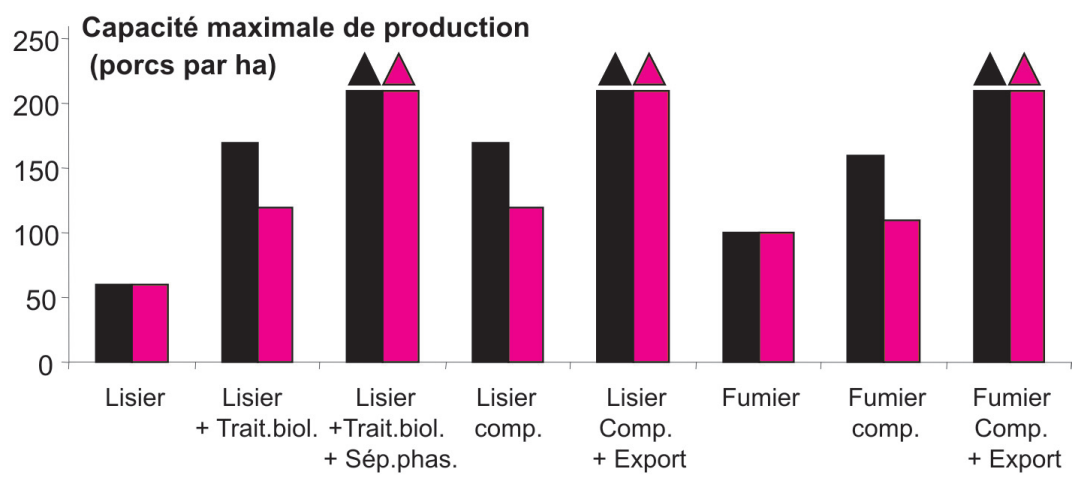

ments potentiellement polluants produits dans les déjections et émis lors des phases de stockage et/ou de traitement des effluents sont calculées sur la base de résultats acquis dans le programme «Porcherie verte», en particulier les facteurs d'émissions, et des recommandations du CORPEN (2003). Concernant la fertilisation, la gestion des apports en azote, en phosphore et en potassium se base sur les règles établies par le COMIFER (1996, 1997).

L'optimisation du fonctionnement de l'exploitation est réalisée en cherchant à maximiser la marge brute de l'exploitation (somme des marges brutes des productions végétales et animales), sous deux hypothèses de contraintes environnementales, l'une portant uniquement sur la quantité d'azote organique $(<170$ unités par hectare de Surface Potentiellement Epandable (SPE)), la deuxième $y$ ajoutant une limite d'apport de phosphore $\left(<100\right.$ unités de $\mathrm{P}_{2} \mathrm{O}_{5}$ par ha de $\left.\mathrm{SAU}\right)$.

Huit filières de gestion des effluents sont comparées en fonction de la quantité de porcs produits à l'ha. Dans cinq d'entre elles, les animaux sont élevés sur caillebotis intégral, ce qui conduit à la production d'un effluent liquide (lisier). Dans les trois autres, les animaux sont élevés sur litières accumulée de paille, ce qui conduit à la production d'un effluent solide (fumier) :

- «Lisier» : le lisier est épandu en l'état sur les SPE de l'exploitation ;

- «Lisier + trait.biol.» : le lisier fait l'objet d'un traitement biologique dans un réacteur (Béline et al 2003) ; Le liquide résultant est épandu sur la SPE de l'exploitation ;

- «Lisier + trait.biol. + Sép. phas.» : une séparation poussée des phases solides et liquides du lisier est effectuée. La phase solide est exportée hors de l'exploitation. La phase liquide est traitée comme précédemment et le liquide résultant est épandu sur la SPE de l'exploitation ;

- «Lisier comp.» : le lisier est composté sur paille (Levasseur 2003) et le compost résultant est épandu sur la SPE de l'exploitation ;

- «Lisier comp. + Export» : le lisier est composté comme précédemment mais tout ou partie du compost résultant est exporté hors de l'exploitation ;

- «Fumier» : le fumier est épandu en l'état sur la SPE de l'exploitation ;

- «Fumier comp.»: le fumier est composté et le compost résultant est épandu sur la SPE de l'exploitation ; 
Figure 11. Bilan environnemental de l'exploitation pour différentes filières de gestion des effluents, sous deux hypothèses de contraintes règlementaires ( $\mathbf{\square}$ limite 170 UN/ha SPE; limites 170 UN/ha SPE et $100 \mathrm{UP}_{2} \mathrm{O}_{5} /$ ha $\left.\mathrm{SAU}\right)$. Les bilans sont calculés sur la base de la capacité maximale de production de porc calculée précédemment (Figure 10). Le bilan apparent de l'azote et du phosphore résulte de la différence entre les entrées et les sorties de l'exploitation (d'après Baudon et al 2005).
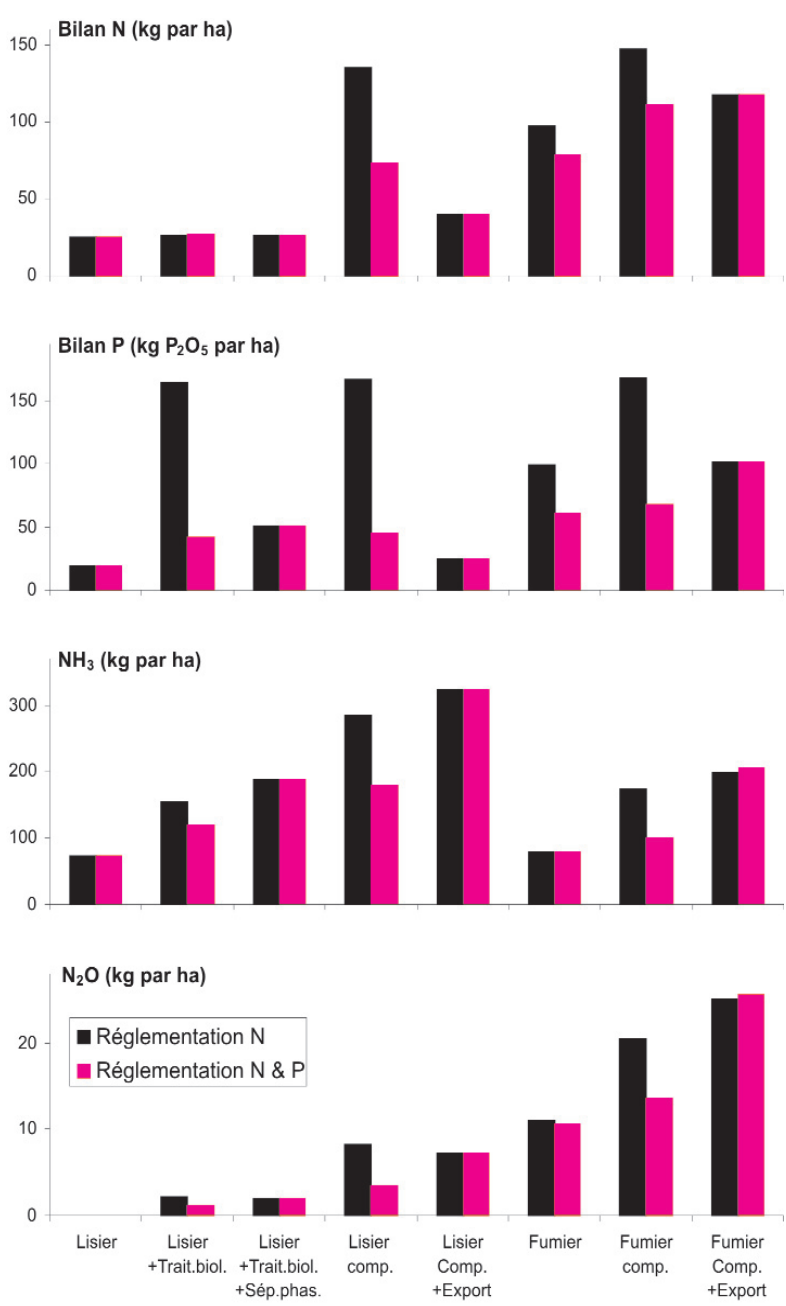

- «Fumier comp. + Export»: le fumier est composté comme précédemment mais tout ou partie du compost résultant est exporté hors de l'exploitation.

Les résultats présentés concernent :

- la capacité maximale de production à l'ha (figure 10);

- les bilans apparents de l'azote et du phosphore (total des entrées dans l'exploitation diminué du total des sorties) exprimés par ha (figures 11 et 12) ;

- les émissions de $\mathrm{NH}_{3}$ et de $\mathrm{N}_{2} 0$ à l'ha (figures 11 et 13 ) ;
Figure 12. Bilan apparent de $N$ et de $P$ en fonction du nombre de porcs produits à l'hectare de SAU, pour différentes filières de gestion des effluents. Les valeurs sont calculées en l'absence de contrainte environnementale sur le phosphore (d'après Baudon et al, 2005).
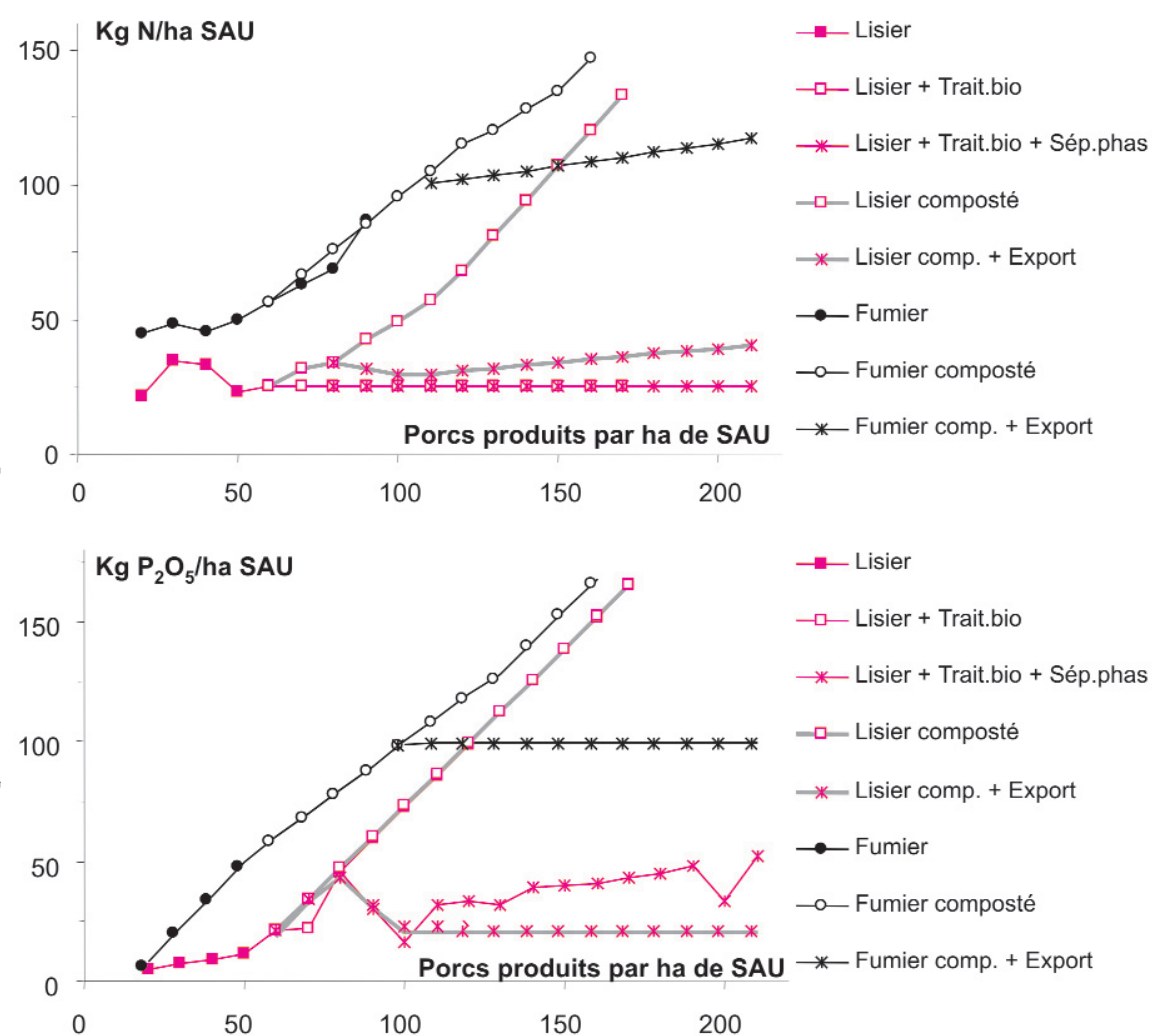

Figure 13. Emissions de gaz azotés en fonction du nombre de porcs produits à l'hectare de SAU, pour différentes filières de gestion des effluents. Les valeurs sont calculées en l'absence de contrainte environnementale sur le phosphore (d'après Baudon et al 2005).
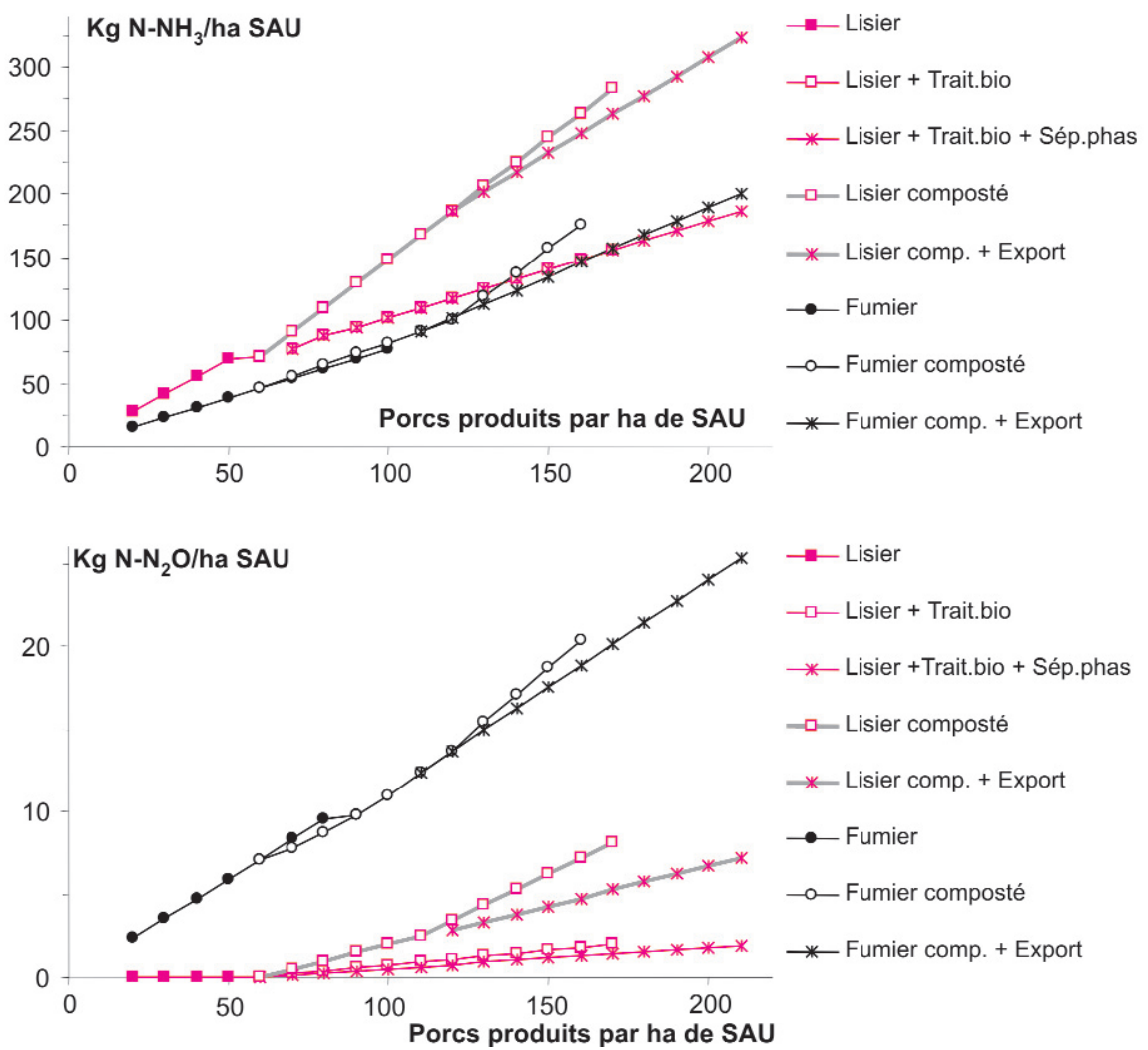
Figure 14. Marge brute d'exploitation en fonction du nombre de porcs produits à l'hectare de SAU, pour différentes filières de gestion des effluents. L'accroissement marginal de la marge brute est calculé en dérivant les équations des courbes décrivant la marge brute. Les valeurs sont calculées en l'absence de contrainte environnementale sur le phosphore (d'après Baudon et al 2005).
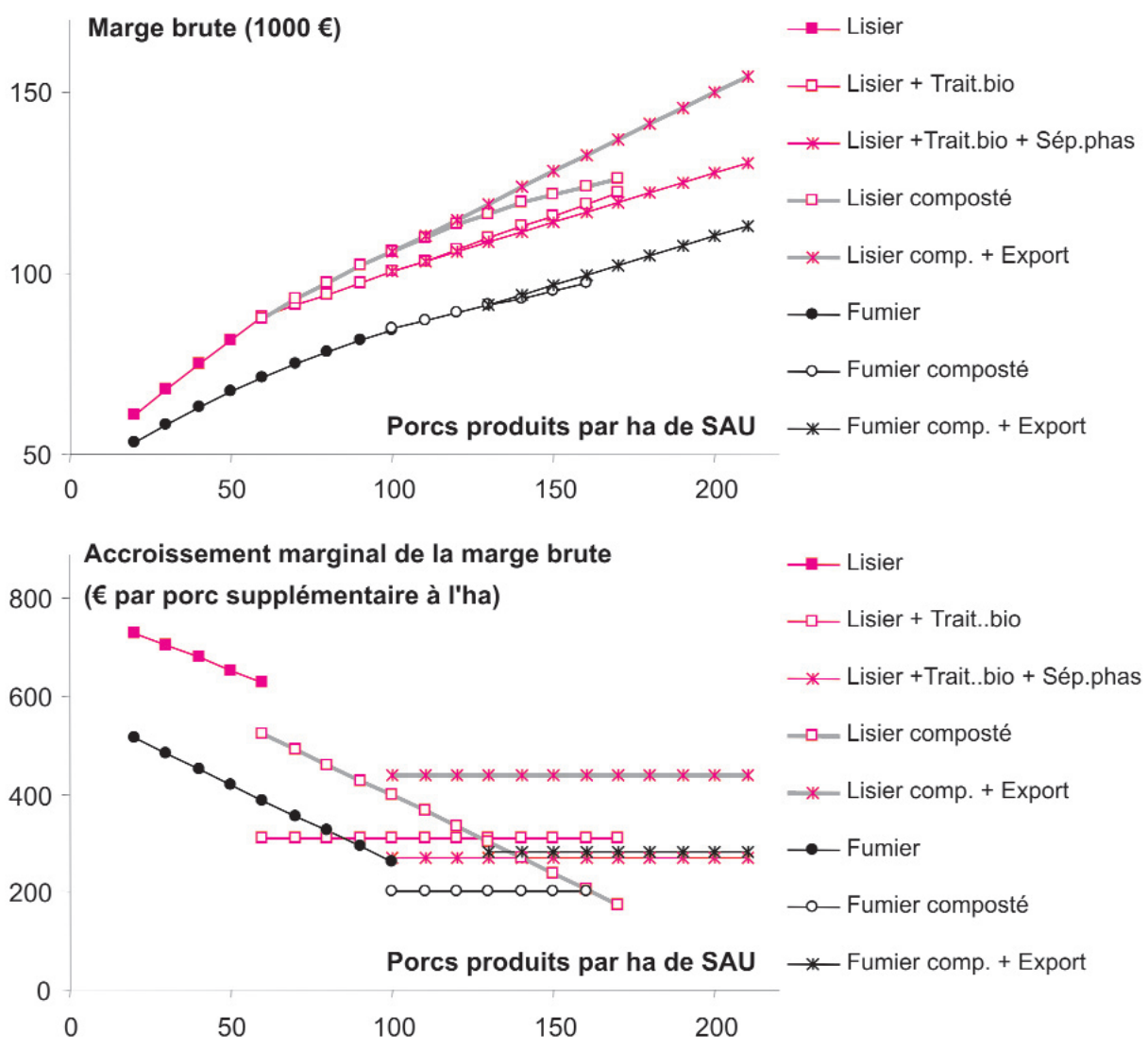

- la marge brute de l'exploitation et l'accroissement marginal de marge brute par porc supplémentaire produit à l'ha (figure 14).

La figure 15, construite avec des valeurs normalisées pour chacune des variables, permet de comparer directement les différentes filières lorsqu'elles sont utilisées au maximum de capacité de production qu'elles permettent sur l'exploitation.

\section{1 / La filière «Lisier brut»}

La filière "Lisier brut» conduit à la meilleure marge brute marginale, avec potentiellement de bons bilans environnementaux, sous réserve de limiter la production à 60 porcs à l'ha.

La filière «Lisier brut» consiste à épandre le lisier en l'état sur les surfaces potentiellement épandables de l'exploitation. En l'absence d'exportation d'effluents, la capacité maximale de production est limitée à 60 porcs à l'ha si les effluents sont sous forme de lisier intégralement épandu sur l'exploitation cette filière suppose aussi que tout soit mis en œuvre pour limiter les nuisances olfactives.

Pour produire plus de 60 porcs à l'ha on peut :

- rester en système lisier mais lui faire subir un traitement (traitement biologique avec ou sans séparation de phases préalable, compostage), visant à diminuer les teneurs en éléments à risque ;

- exporter du lisier ou des coproduits hors de l'exploitation. Compte tenu de sa richesse en eau, le lisier n'est exportable dans des conditions économiquement acceptables que sur des exploitations voisines ;

- mettre les animaux sur litière.

\section{2 / Les filières «Traitement biologique du lisier»}

a) Le traitement biologique du lisier sans séparation de phases permet d'augmenter la production de porcs mais détériore le bilan apparent de phosphore de l'exploitation et réduit significativement la marge brute par porc produit

Le traitement biologique simple, sans séparation de phases, d'une partie ou de la totalité du lisier réduit sensiblement la quantité d'azote des effluents mais pas celle de phosphore (cf. article sur le devenir des éléments à risque). Il permet d'augmenter notablement la capacité de production porcine de l'exploitation, jusqu'à un maximum de 170 porcs à l'ha en l'absence de contrainte environnementale sur le phosphore (figure 10). Cette capacité est réduite à 120 porcs à l'ha avec la contrainte phosphore. Le bilan apparent de l'azote reste aussi bon que dans le cas du lisier non traité, mais le bilan apparent du $\mathrm{P}$ est fortement détérioré en l'absence de contrainte sur le P (figures 11 et 12). Les émissions gazeuses de $\mathrm{NH}_{3}$ sont accrues, principalement du fait de l'augmentation des émissions dans le bâtiment en relation avec l'accroissement du nombre de porcs produits, de même que celles de $\mathrm{N}_{2} \mathrm{O}$, du fait des émissions associées au traitement biologique. L'augmentation de la production porcine permet d'accroître la marge brute de l'exploitation (figure 14), mais le traitement du lisier excédentaire grève fortement les coûts, ce qui fait que l'accroissement marginal de la marge brute par porc supplémentaire produit est nettement plus faible qu'en l'absence de traitement (cf. aussi la figure 15b). 
b) La mise en cuvre d'un procédé de séparation de phases préalablement au traitement biologique du lisier permet d'augmenter encore la production de porcs en respectant des contraintes environnementales sur le phosphore, mais il réduit encore plus la marge brute par porc produit

$\mathrm{Du}$ fait que la majeure partie du phosphore des lisiers est associé aux fractions solides, la mise en œuvre d'une séparation des phases solides et liquides préalablement au traitement biologique du lisier permet de réduire sensiblement les quantités de phosphore à épandre sur l'exploitation, sous réserve de pouvoir exporter les coproduits issus du procédé (cf. article sur le devenir des éléments à risque). La capacité maximale de production passe alors à 210 porcs par ha (figure 10). Le bilan apparent de $\mathrm{N}$ dans l'exploitation reste faible et celui du $\mathrm{P}$ atteint $50 \mathrm{~kg}$ par ha de SAU, soit 2,5 fois celui observé avec la filière «Lisier brut» (figures 11 et 12). Les émissions gazeuses de $\mathrm{NH}_{3}$ et de $\mathrm{N}_{2} \mathrm{O}$ restent du même ordre de grandeur qu'en l'absence de séparation de phases (figures 11 et 13). L'augmentation de la production porcine permet d'accroître encore la marge brute de l'exploitation (figure 14), mais la mise en œuvre de la séparation de phases ajoute un coût supplémentaire qui grève plus lourdement les coûts de production, conduisant à un affaiblissement encore plus marqué de l'accroissement marginal de la marge brute par porc supplémentaire produit (cf. aussi la figure $15 \mathrm{~b})$.

\section{3 / Les filières «Compostage du lisier»}

a) Le compostage du lisier peut être une alternative au traitement biologique pour les petites exploitations, mais son bilan environnemental est médiocre

La mise en œuvre du traitement biologique du lisier entraîne un investissement lourd qui n'est pas forcément accessible aux exploitations de taille faible ou moyenne. Le compostage du lisier sur paille constitue alors une alternative qui permet d'abattre une partie importante de l'azote des effluents, mais n'a aucun effet sur le phosphore (cf. article sur le devenir des éléments à risque). La capacité maximale de production est similaire à celle obtenue avec un traitement biologique sans séparation de phases (figu- re 10). Les bilans apparents de l'azote et du phosphore (figures 11 et 12) augmentent sensiblement par rapport à ce qui est observé avec un traitement biologique. En effet, du fait de la faible disponibilité à court terme de l'azote du compost, celui-ci ne peut servir de fertilisant pour les cultures de céréales au printemps, et il reste donc nécessaire d'acheter des engrais minéraux. Les émissions gazeuses de $\mathrm{NH}_{3}$ et de $\mathrm{N}_{2} \mathrm{O}$ sont aussi fortement accrues (figures 11 et 13 (cf. article sur les émissions gazeuses). La mise en œuvre du compostage représente un investissement modeste mais coûte cher en fonctionnement (achats d'intrants et travail). C'est ce qui explique que l'accroissement marginal de marge brute par porc supplémentaire produit est supérieur à celui obtenu avec le traitement biologique pour les chargements modérés (moins de 100-120 porcs à l'ha) mais inférieur pour les chargements élevés, au-delà de 130140 porcs à l'ha (figure 14, cf. aussi la figure $15 \mathrm{c})$.

b) L'exportation $d u$ compost de lisier allège les bilans apparents de $N$ et de $P$ de l'exploitation mais ne change pas les émissions gazeuses. Il permet de maintenir une bonne marge brute, même aux chargements élevés

L'exportation de compost hors de l'exploitation permet d'augmenter sensiblement la capacité maximale de production qui peut dépasser 210 porcs à l'ha (figure 10). L'exportation du compost permet d'alléger sensiblement les bilans apparents de l'azote et du phosphore et de les ramener à des niveaux similaires à ceux observés pour les filières de traitement biologique (figures 11 et 12). Reste toutefois à vérifier que cette exportation n'alourdit pas excessivement les bilans des sites d'accueil du compost. Les émissions gazeuses de $\mathrm{NH}_{3}$ et de $\mathrm{N}_{2} \mathrm{O}$ (figures 11 et 13) restent par contre à des niveaux très élevées (figures 11 et 13) car ces émissions se produisent essentiellement pendant le processus de compostage (cf. article sur les émissions gazeuses). L'exportation du compost permet d'obtenir de bonnes marges brutes, y compris avec des chargements élevés (figure 14), ce qui pourrait rendre cette technique attrayante pour de grosses exploitations, sous réserve de résoudre la logistique de l'approvisionnement en paille et de trouver un débouché pour le compost (cf. aussi la figure $15 \mathrm{c})$.

\section{4 / Les filières «Fumier»}

a) L'élevage de porcs sur litière permet de produire plus de porcs à l'ha mais au prix d'une dégradation de l'impact environnemental et d'une augmentation des coûts de production

Comparativement à la filière «Lisier brut», la filière «Fumier» qui consiste à loger les animaux sur litière de paille, permet d'accroître la capacité maximale de production de porcs jusqu'à 100 porcs à l'ha (figure 10), grâce au fait qu'une partie importante de l'azote excrété par les animaux est volatilisé par les fermentations de la litière (cf. article sur le devenir des éléments à risque). Les bilans de l'azote et du phosphore au niveau de l'exploitation sont dégradés (figures 11 et 12) car il reste nécessaire d'acheter des engrais minéraux du fait que le compost ne peut pas être épandu directement sur les cultures de céréales au printemps. Les émissions de $\mathrm{NH}_{3}$ sont similaires à celles de la filière «Lisier brut» mais il y a émission de $\mathrm{N}_{2} \mathrm{O}$ (figures 11 et 13 , cf. article sur les émissions gazeuses). Parce que les coûts de production sont plus élevés avec la filière «Litière», il faut produire 100 porcs à l'ha pour obtenir la même marge brute d'exploitation qu'avec 50 porcs à l'ha en filière «Lisier brut» (figure 14, cf. aussi la figure $15 \mathrm{a}$ ). Ce constat doit cependant être nuancé par le fait que ce mode de production présente les avantages de la litière en matière de nuisances et de bien-être animal (cf. §1) et d'amélioration à long terme de la structure des sols. Par ailleurs, le modèle surestime sur le moyen-long terme les effets défavorables dus aux apports d'engrais minéraux. En effet, il ne tient pas compte du fait que ces apports peuvent être réduits au bout de quelques années, dès lors que la minéralisation de l'azote organisé apporté par les fumiers contribue de façon significative au pool d'azote disponible dans le sol (cf : avant dernier $\S$ de la section $1.5 \mathrm{de}$ l'article sur le devenir des éléments à risques).

b) Le compostage du fumier des litières permet d'augmenter la production de porcs à l'ha, mais uniquement en l'absence de contrainte environnementale sur le phosphore, et l'accroissement marginal de marge brute est faible

Le compostage des fumiers permet de réduire encore plus la quantité d'azote, sans aucun effet sur le phosphore. La capacité maximale de production s'accroît alors de 100 à 
Figure 15. Récapitulatif des résultats obtenus par simulation de huit filières de gestion des effluents d'élevage au niveau d'une exploitation. Capacité : capacité maximale de production de porc à l'hectare ; Bilan N : quantité d'azote excédentaire à l'hectare ; Bilan $P$ : quantité de phosphore excédentaire à l'hectare ; $\mathrm{N} 2 \mathrm{O}$ : émissions de $\mathrm{N}_{2} \mathrm{O}$ à l'hectare ; Marge : marge brute de l'exploitation. Les valeurs sont calculées en l'absence de contrainte environnementale sur le phosphore et pour un nombre de porcs produits égal à la capacité maximale de production. Pour faciliter la comparaison, chaque variable a été normalisée entre 0 et 1 (valeurs recalculées d'après Baudon et al 2005).

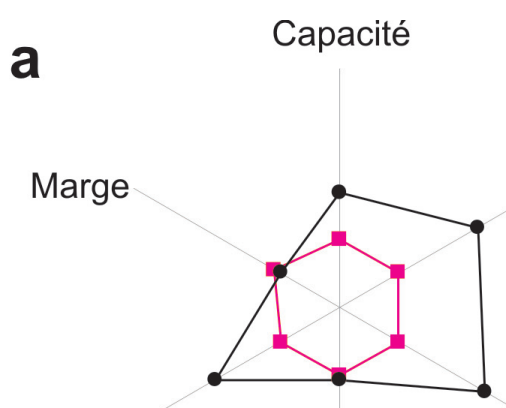

$\mathrm{N} 20$

Bilan P Marge

\section{Capacité}

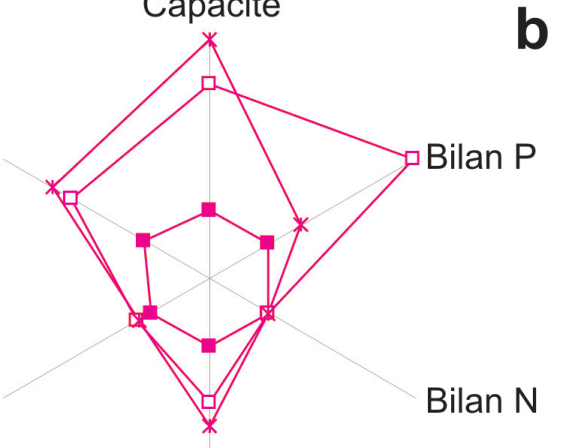

b

Bilan N

N20

$\rightarrow$ Lisier

$\mathrm{NH} 3$

$\rightarrow-$ Lisier + Trait. biol.

* Lisier +T.bio. +Sép. pha.

- - Lisier composté

* Lisier comp. +Export

$\rightarrow$ Fumier

C

Capacité

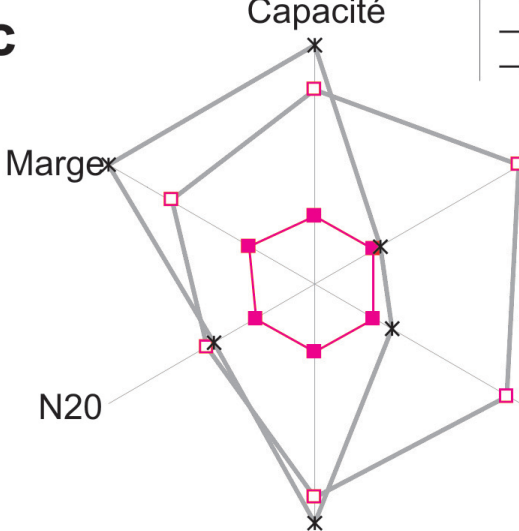

$\mathrm{NH} 3$
- - Fumier composté

*-Fumier comp. +Export
$\mathrm{NH} 3$

Capacité

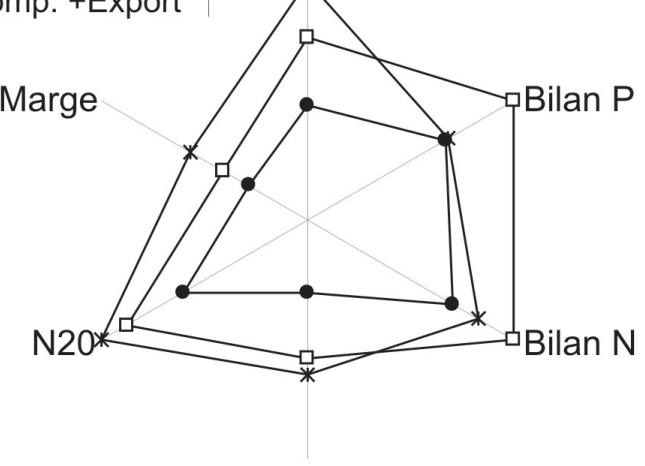

$\mathrm{NH} 3$

e

Capacité

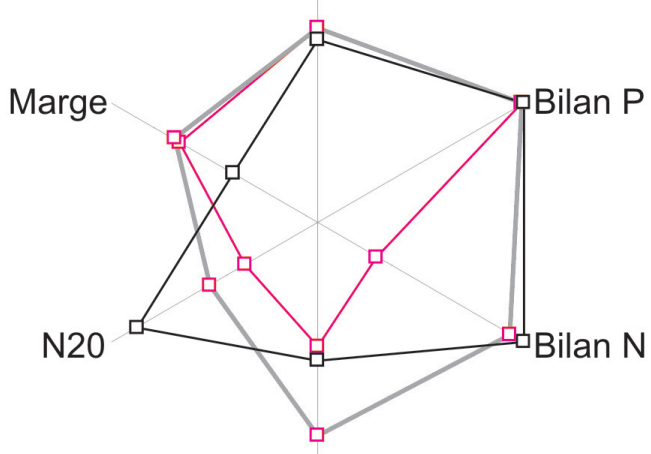

$\mathrm{NH} 3$

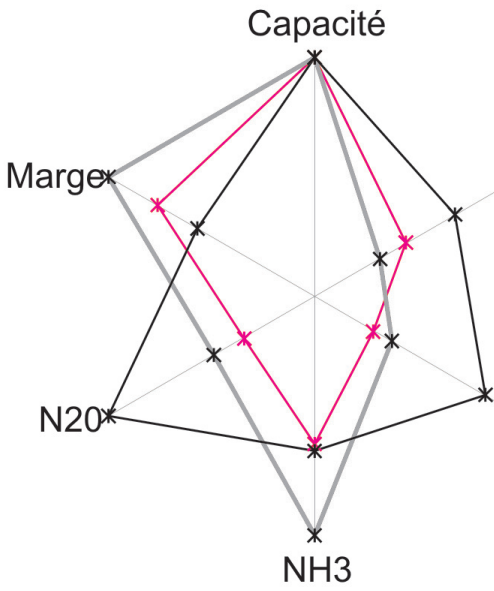

Bilan P

Bilan N

Pour des raisons de clarté, la comparaison des différentes filières est répartie sur 6 figures : a : lisier brut vs fumier ; b : lisier brut vs lisier traité avec ou sans séparation de phase ; c : lisier brut vs lisier composté avec ou sans export hors de l'exploitation ; d : fumier en l'état vs fumier composté avec ou sans export hors de l'exploitation ; e : lisier traité vs lisier composté ou fumier composté, sans export hors de l'exploitation ; f : lisier traité vs lisier composté ou fumier composté, avec export hors de l'exploitation). 
160 porcs à l'ha (figure 10), mais uniquement s'il n'y a pas de contrainte environnementale sur le phosphore. Le bilan environnemental local s'en trouve alors fortement dégradé (figures 11 et 12), aussi bien en termes de bilans $\mathrm{N}$ et $\mathrm{P}$ de l'exploitation que d'émissions gazeuses de $\mathrm{NH}_{3}$ et de $\mathrm{N}_{2} \mathrm{O}$ (figures 11 et 13). L'accroissement d'effectif (en l'absence de contrainte sur le P) permet d'augmenter la marge brute de l'exploitation mais de façon peu efficiente (figure 14). Cette filière conduit en effet au plus faible accroissement marginal de marge brute par porc supplémentaire produit (cf. aussi la figure $15 \mathrm{~d}$ ).

c) L'exportation du compost excédentaire permet d'augmenter encore la production de porcs en allégeant les bilans apparents de $N$ et $P$, mais pas les émissions gazeuses

Le compostage des fumiers peut avoir un intérêt par le fait que le compost est plus facilement exportable en dehors des exploitations. La capacité maximale de production peut alors dépasser 210 porcs à l'ha (figure 10). L'exportation permet d'alléger un peu les bilans apparents de l'azote et du phosphore de l'exploitation, qui restent néanmoins à des niveaux élevés (figures 11 et 12), alors que les émissions gazeuses s'accroissent en même temps que le niveau de production de porcs, et atteignent des niveaux tout particulièrement élevés dans le cas du $\mathrm{N}_{2} \mathrm{O}$ (figures 11 et 13). L'accroissement d'effectif permet d'augmenter la marge brute de l'exploitation (figure 14) avec une efficacité similaire à celle observée avec les filières de traitement biologique (cf. aussi la figure 15d).

2.5 / Parmi les filières avec traitement, qui autorisent des chargements élevés, le traitement biologique semble réaliser le meilleur compromis entre marge brute réalisée et impact sur l'environnement, si l'on néglige les transferts d'impact hors de l'exploitation

Les filières comportant un traitement des effluents sans exportation (lisier + traitement biologique, lisier composté, fumier composté) autorisent des niveaux similaires de capacité maximale de production (160-170 porcs par ha) et ont toutes le même bilan phosphore (figure 15e). Pour le reste, des différences importantes apparaissent entre filières :

- le traitement biologique du lisier est plus efficace pour réduire les rejets et émissions de composés azotés et il autorise une bonne marge brute ;

- le compostage du fumier conduit à une marge brute plus faible que le traitement biologique, et s'accompagne d'une détérioration sensible du bilan d'azote et d'un accroissement des émissions de $\mathrm{N}_{2} \mathrm{O}$;

- le compostage du lisier conduit à la même marge brute que le traitement biologique mais au prix d'une détérioration sensible du bilan d'azote et d'émissions accrues de $\mathrm{N}_{2} \mathrm{O}$.

Pour des chargements de l'ordre de 170 porcs à l'ha, le traitement biologique semble réaliser le meilleur compromis entre marge brute réalisée et impact sur l'environnement. Cette conclusion doit cependant être nuancée par le fait que le modèle ne prend pas en compte les impacts environnementaux extérieurs à l'exploitation, en particulier la production de l'énergie nécessaire au traitement biologique. Par ailleurs, le modèle ne prend pas en compte les avantages de la litière en matière de nuisances et de bien-être animal (cf. §1).

\section{6 / Parmi les filières avec trai- tement et exportation, qui per- mettent des niveaux de charge- ment très élevés, le compostage de fumier semble cumuler les inconvénients en termes de mar- ge brute et d'impacts environne- mentaux, si l'on néglige les transferts d'impact hors de l'ex- ploitation associés au traitement biologique}

Les filières comportant un traitement des effluents avec exportation de coproduits (lisier + traitement biologique + séparation de phases, lisier composté + export, fumier composté + export) autorisent des niveaux similaires de capacité maximale de production, supérieurs à 210 porcs à l'ha (figure 15f). Pour le reste, des différences importantes apparaissent entre filières :

- le traitement biologique du lisier avec séparation de phases, conduit à des bilans corrects de $\mathrm{N}$ et de $\mathrm{P}$ avec des émissions faibles de $\mathrm{N}_{2} \mathrm{O}$ et des émissions assez élevés de $\mathrm{NH}_{3}$. La marge brute obtenue est intermédiaire entre celles des deux autres filières ;

- le compostage du fumier avec exportation conduit à des impacts environnementaux similaires $\left(\mathrm{NH}_{3}\right)$ ou nettement plus élevés (bilans $\mathrm{N}$ et $\mathrm{P}, \mathrm{N}_{2} \mathrm{O}$ ) en dégageant une marge brute sensiblement plus faible ;
- le compostage du lisier conduit à la meilleure marge brute avec des impacts environnementaux globalement un peu moins bons que le traitement biologique.

Là encore, le relatif bon positionnement du traitement biologique serait à nuancer par la prise en compte des impacts environnementaux externes et le mauvais positionnement des litières par leurs avantages sur les dimensions «nuisances» et «bien-être» (cf. §2.5).

\section{7 / Interactions entre produc- tion porcine et cultures au sein de l'exploitation}

La figure 16 illustre les interactions entre la production porcine et les cultures mises en place au sein de l'exploitation. L'exemple considéré ici est une exploitation utilisant la filière lisier avec compostage de l'excédent produit par les porcs produits au-delà de 60 porcs à l'ha. Rappelons que le modèle cherche à optimiser la marge brute dégagée sur l'exploitation entière, en fonction du nombre de porcs produits à l'ha :

- pour des chargements très faibles, (20 porcs à l'ha), la quantité d'effluent à épandre est inférieure aux capacités d'accueil des surfaces consacrées aux cultures. Il est donc possible d'introduire des légumineuses dans l'assolement (figure 16a) et l'exploitation peut assurer elle-même $80 \%$ de son approvisionnement en matières premières pour l'alimentation des porcs (figure 16b), y compris en termes de ressources protéiques (pois et colza) ;

- à partir de 30 porcs à l'ha, les légumineuses disparaissent de l'assolement. Jusqu'à 50 porcs à l'ha, il est encore possible de cultiver du colza qui peut contribuer à l'approvisionnement en protéines des animaux. Le taux d'autonomie pour l'alimentation des porcs reste élevé ( $>70 \%)$ et l'autonomie en matières de fertilisation grimpe rapidement jusqu'à $65 \%$;

- au-delà de 60 porcs à l'ha, le colza disparaît à son tour de l'assolement pour laisser totalement la place aux céréales dont la paille est nécessaire au compostage du lisier excédentaire. Le taux d'autonomie en matière d'alimentation des animaux chute rapidement alors que l'autonomie pour la fertilisation se maintient aux alentours de 70$80 \%$;

- au-delà de 100 porcs à l'ha, l'exploitation n'est plus totalement autonome pour la fourniture de la paille nécessaire au compostage. Les achats de 
Figure 16. Interactions entre intensité de la production porcine (nombre de porcs produits à l'hectare) et assolement des cultures dans une exploitation épandant la totalité du lisier produit sur sa SAU propre. Pour les charges supérieures à 50 porcs à l'hectare, une part croissante du lisier est composté sur paille (d'après Dourmad et al 2005, 2006).

a. Assolement.

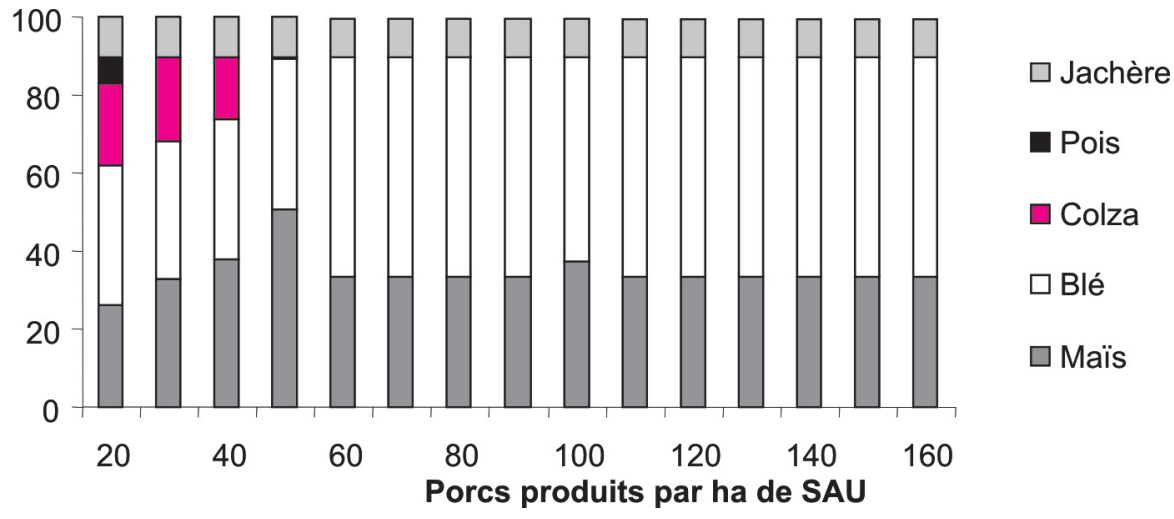

b. Degrés d'autonomie pour la fertilisation et la fourniture d'aliment et de paille.

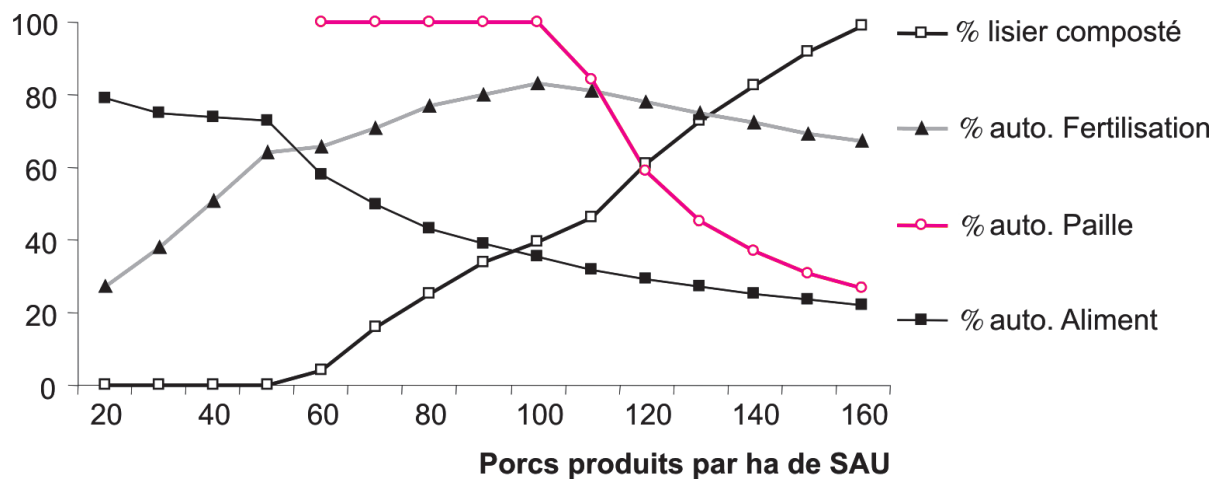

paille risquent alors de grever fortement les coûts de production.

\section{8 / Les limites du modèle}

Le travail réalisé dans le cadre du programme «Porcherie verte» ne constituait qu'une première étape du développement du modèle d'exploitation qui a vocation à s'enrichir via la prise en compte des autres phases de la production porcine, «maternité» et «post-sevrage» et une plus grande sophistication de la description des cultures sur l'exploitation. Les calculs économiques devront aussi être affinés, en particulier en prenant en compte les effets d'échelle sur les coûts de traitement et d'épandage. Mais il y a aussi d'autres limites intrinsèques qu'il convient de souligner :

- les calculs économiques ne prennent pas en considération la possibilité de mieux valoriser les porcs produits sur litière, par exemple au travers d'un label de qualité ;

- les émissions gazeuses sont calculées à partir de valeurs moyennes. On sait pourtant qu'elles peuvent être extrêmement variables, en particulier en fonction du mode de conduite des litières. Pour la filière «Fumier», les émissions de $\mathrm{NH}_{3}$ calculées représentent environ $13 \%$ de l'azote excrété et se situent donc légèrement en-dessous de la fourchette indiquée par l'équipe de Robin et Hassouna (15-25\%, cf. tableau 2 de l'article sur les émissions gazeuses). Pour cette même filière, les émissions de $\mathrm{N}_{2} \mathrm{O}$, estimées à $1,9 \%$ de l'azote excrété, se situent nettement en dessous de la fourchette indiquée par ces mêmes auteurs (4 à 12\%). La production de gaz à effet de serre des filières fumier est donc vraisemblablement sous-évaluée ;

- le modèle se limite aux flux des principaux éléments à risque pour l'environnement sans prendre en compte d'autres composantes essentielles de l'acceptabilité sociale de la production porcine comme le bien-être animal ou les mauvaises odeurs (cf. §1). Leur prise en compte interviendrait lourdement dans la comparaison des filières «Lisier» et «Fumier», au bénéfice de ces dernières ;
- le modèle se limite aux impacts environnementaux exprimés au niveau de l'exploitation. Les impacts environnementaux délocalisés (par exemple les gaz à effet de serre générés par la production de l'énergie nécessaire au traitement biologique) ne sont pas pris en compte. Les travaux exposés au paragraphe 3 de cet article visent à prendre en compte les impacts environnementaux de façon beaucoup plus globale, tout au long de la chaîne de production ;

- le modèle génère des assolements optimisés selon leur aptitude à fournir alimentation, paille et surfaces d'épandage. Les contraintes agronomiques de rotation ne sont pas prises en compte alors qu'elles sont décisives du bon fonctionnement des systèmes de culture, notamment dans le domaine de la protection des cultures, générateur éventuel d'autres impacts environnementaux liés à l'usage des pesticides. De même, l'impact de ces assolements très simplifiés (jachère, céréales, maïs) sur la biodiversité n'est pas abordé.

\section{3 / Evaluation globale de l'impact environnemental des élevages porcins}

\section{1 / L'analyse de cycle de vie est une méthode de choix pour l'a- nalyse globale de l'impact envi- ronnemental de l'élevage porcin}

Il existe de nombreuses méthodes pour évaluer l'impact environnemental des activités agricoles. Les analyses concentrées sur un seul problème (par exemple la pollution de l'eau par les nitrates) peuvent conduire à des transferts de pollution du seul compartiment cible considéré (par exemple l'eau) vers un autre compartiment de l'environnement qui n'est pas pris en compte (par exemple 1'atmosphère). Par ailleurs les études réalisées à une échelle restreinte (élevage porcin ou exploitation) peuvent aussi conduire à des transferts de pollution à l'extérieur des frontières de l'entité prise en compte. Il est donc impératif d'utiliser des méthodes globales, multicritères, qui prennent en compte simultanément l'ensemble des impacts environnementaux identifiés, trouvant leur origine dans les divers éléments de la chaîne de production et affectant l'ensemble des compartiments de l'environnement susceptibles de l'être.

Une première analyse comparative de 12 méthodes basées sur des indicateurs 
Tableau 3. Principales caractéristiques globales de cinq méthodes d'évaluation environnementale des activités agricoles (d'après van der Werf et al 2007).

\begin{tabular}{|c|c|c|c|c|c|}
\hline Sigle & ACV & EE & EMA & FarmSmart & DIALECTE \\
\hline Nom anglais & $\begin{array}{c}\text { Life Cycle } \\
\text { Analysis (LCA) }\end{array}$ & $\begin{array}{l}\text { Ecological } \\
\text { Footprint }\end{array}$ & $\begin{array}{c}\text { Environmental } \\
\text { Management } \\
\text { for Agriculture }\end{array}$ & FarmSmart & 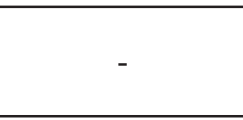 \\
\hline Nom français & $\begin{array}{l}\text { Analyse du } \\
\text { Cycle de Vie }\end{array}$ & $\begin{array}{l}\text { Empreinte } \\
\text { écologique }\end{array}$ & $\begin{array}{c}\text { Gestion } \\
\text { environnementale } \\
\text { de l'agriculture }\end{array}$ & - & DIALECTE \\
\hline $\begin{array}{c}\text { Objectifs } \\
\text { Globaux } \\
\text { d'évaluation }\end{array}$ & $\begin{array}{c}\text { Impact } \\
\text { environnemental }\end{array}$ & $\begin{array}{c}\text { Impact } \\
\text { environnemental }\end{array}$ & $\begin{array}{c}\text { Adhésion } \\
\text { bonnes pratiques }\end{array}$ & $\begin{array}{c}\text { Impact } \\
\text { environnemental }\end{array}$ & $\begin{array}{c}\text { Impact } \\
\text { environnemental }\end{array}$ \\
\hline $\begin{array}{c}\text { Types } \\
\text { d'objectifs }\end{array}$ & $\begin{array}{l}\text { Intrants, } \\
\text { Emissions } \\
\end{array}$ & $\begin{array}{l}\text { Intrants, } \\
\text { Emissions }\end{array}$ & $\begin{array}{l}\text { Pratiques, } \\
\text { Emissions }\end{array}$ & $\frac{\text { Intrants }}{\text { Emissions }}$ & $\begin{array}{c}\text { Pratiques, } \\
\text { Intrants, } \\
\text { Etat système } \\
\end{array}$ \\
\hline $\begin{array}{l}\text { Nombre } \\
\text { d'objectifs }\end{array}$ & 6 & 3 & 12 & 6 & 19 \\
\hline
\end{tabular}

et se limitant à l'échelle de l'exploitation (van der Werf et Petit 2002) a permis d'élaborer un certain nombre de recommandations :

- prendre en compte une gamme d'impacts environnementaux qui permette de couvrir à la fois des effets locaux et globaux ;

- préférer les indicateurs mesurant l'effet environnemental des pratiques à ceux qui se contentent de constater l'adhésion ou non à une pratique ;

- préférer les indicateurs qui permettent de calculer les impacts à la fois par hectare de surface utilisée et par kg de porc produit. Le calcul par hectare

Tableau 4. Caractéristiques des trois scénarios de production étudiés par Basset-Mens et Van der Werf $(2004,2005)$ et van der Werf et al (2007).

\begin{tabular}{|l|c|c|c|}
\hline & BPA & LR & AB \\
\cline { 2 - 4 } & $\begin{array}{c}\text { Bonnes } \\
\text { Pratiques } \\
\text { Agricoles }\end{array}$ & $\begin{array}{c}\text { Label } \\
\text { Rouge }\end{array}$ & $\begin{array}{c}\text { Agriculture } \\
\text { Biologique }\end{array}$ \\
\hline Production de porcelets & & & \\
Logement & Caillebotis & Plein air & Plein air \\
Porcelets sevrés/truie productive/an & 25,5 & 22,6 & 20,3 \\
Age au sevrage, jours & 25,7 & 28 & 42 \\
Surface par truie, m2 & $<4$ & 1000 & 1000 \\
Aliment par truie (verrats inclus), kg/an & 1313 & 1490 & 1695 \\
\hline Sevrage/vente & & & \\
Logement & Caillebotis & Litière de & Litière de \\
& & paille & paille \\
Surface par porc, m ${ }^{2}$ & 0,85 & 2,6 & 2,3 \\
Indice de consommation & 2,7 & 2,9 & 3,2 \\
Age à l'abattage, jours & 175 & 190 & 195 \\
Poids à l'abattage, kg $\mathrm{kg}$ & 113 & 115 & 120 \\
Aliment consommé, kg & 275 & 312 & 340 \\
\hline
\end{tabular}

est le plus pertinent pour les effets locaux/régionaux comme la toxicité terrestre ou l'eutrophisation. Il n'a par contre techniquement pas de sens (même s'il peut être utilisé sur le plan politique en termes de négociations entre pays) pour les effets globaux se manifestant à l'échelle du globe, comme par exemple le changement climatique induit par les gaz à effet de serre, pour lesquels on lui préfèrera le calcul par kg de produit;

- préférer les indicateurs qui donnent des valeurs continues à ceux exprimés par des notes ;

- établir, sur une base scientifique, des valeurs seuils pour les indicateurs, qui correspondent à des points singuliers dans la réponse de l'environnement à l'impact considéré.

Dans un deuxième temps, cinq méthodes (tableau 3) ont été utilisées pour classer trois systèmes de production porcine contrastés (tableau 4) dont les impacts environnementaux sont a priori très différents. Les résultats de ce travail (van der Werf et al 2007) montrent que les résultats obte-

Tableau 5. Comparaison des évaluations de trois scénarios de production porcine (cf. tableau 4) selon cinq méthodes (cf. tableau 3). Pour chaque méthode les scénarios sont classés de 1 (le plus favorable) à 3 (le plus défavorable) (d'après Van der Werf et al 2007).

\begin{tabular}{|c|c|c|c|c|c|c|c|c|}
\hline \multicolumn{2}{|c|}{ Méthodes } & \multirow[b]{2}{*}{ ACV } & \multirow[b]{2}{*}{ EE } & \multicolumn{2}{|c|}{ EMA } & \multirow{2}{*}{$\begin{array}{l}\text { Farm } \\
\text { Smart }\end{array}$} & \multicolumn{2}{|c|}{ Dialecte } \\
\hline $\begin{array}{c}\text { Mode } \\
\text { d'expression }\end{array}$ & Scénarios & & & Performance & Emissions & & $\begin{array}{l}\text { Indicateurs } \\
\text { agro- } \\
\text { écologiques }\end{array}$ & $\begin{array}{l}\text { Approche } \\
\text { ferme } \\
\text { entière }\end{array}$ \\
\hline \multirow{3}{*}{$\begin{array}{l}\text { Exploitation } \\
\text { dans son } \\
\text { ensemble }\end{array}$} & BPA & & & 1 & & & 2 & 3 \\
\hline & LR & & & 1 & & & 2 & 2 \\
\hline & $\mathrm{AB}$ & & & 1 & & & 1 & 1 \\
\hline \multirow{3}{*}{ Par hectare } & BPA & 3 & 3 & & 3 & 3 & & \\
\hline & LR & 2 & 2 & & 2 & 2 & & \\
\hline & $\mathrm{AB}$ & 1 & 1 & & 1 & 1 & & \\
\hline \multirow{3}{*}{$\begin{array}{l}\text { Par kg de porc } \\
\text { produit }\end{array}$} & BPA & 1 & 1 & & 1 & 2 & & \\
\hline & LR & 2 & 2 & & 1 & 3 & & \\
\hline & $\mathrm{AB}$ & 3 & 3 & & 1 & 1 & & \\
\hline
\end{tabular}


Figure 17. Impacts environnementaux normalisés de trois scénarios contrastés de production porcine (cf. Tableau 4 ; les impacts sont ramenés à $54.4 \mathrm{~kg}$ de porc produit et divisés par l'impact environnemental total par habitant ; d'après Basset-Mens et Van der Werf 2005).

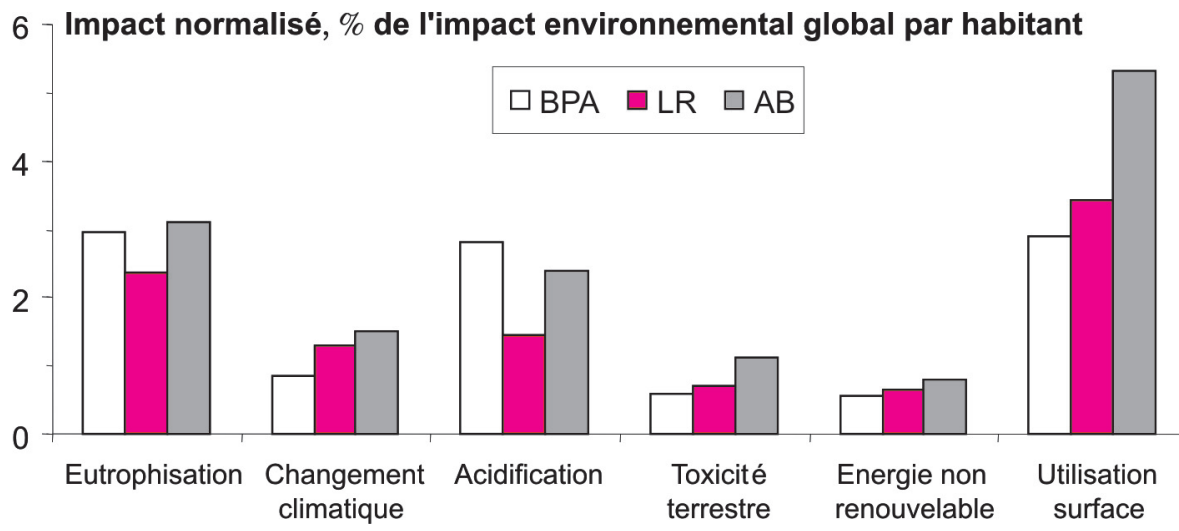

nus peuvent varier selon la méthode et le mode d'expression utilisés (tableau 5). La méthode EMA s'est majoritairement montrée incapable de différencier les trois scénarios. Les différentes méthodes donnent le même classement des impacts lorsqu'ils sont exprimés par hectare mais pas lorsqu'ils sont ramenés au $\mathrm{kg}$ de porc produit.

Les classements des systèmes de production sont inversés selon que l'on exprime les impacts par $\mathrm{kg}$ de porc produit (ce mode d'expression traduit l'efficacité globale de transformation en viande des intrants et s'impose pour les impacts globaux) ou par hectare (ce mode d'expression, le plus pertinent pour les impacts locaux, est très sensible au degré d'intensité des productions).

A l'issue de cette comparaison de méthodes, l'Analyse de Cycle de Vie
(ACV) a été retenue, parce qu'elle repose sur des indicateurs continus, qu'elle prend en compte les phases en amont de l'exploitation et qu'elle repose davantage sur les émissions, qui constituent la cible finale de l'évaluation, que sur les intrants ou les pratiques qui n'en constituent qu'une approche indirecte.

\section{2 / Comparaison des impacts environnementaux de trois scé- narios contrastés de production porcine}

Basset-Mens et van der Werf (2004, 2005) ont utilisé l'analyse du cycle de vie pour évaluer l'impact environnemental de trois scénarios de production porcine contrastés : Bonnes Pratiques Agricoles (BPA), Label Rouge (LR) et Agriculture Biologique (AB) (tableau 4). Les effets environnementaux sont estimés à chacune des étapes de la production, en amont de l'élevage (lors de la production des intrants) et dans l'exploitation. Les résultats cumulés sont intégrés dans sept indicateurs synthétiques correspondant à autant de grandes catégories d'impacts, selon les recommandations de Guinée et al (2002) :

- l'eutrophisation, qui prend en compte les rejets de $\mathrm{NH}_{3}, \mathrm{NO}_{3}, \mathrm{NO}_{2}$, $\mathrm{NO}_{\mathrm{x}}$, et $\mathrm{PO}_{4}$;

- le changement climatique, qui prend en compte les émissions de gaz à effet de serre, $\mathrm{CO}_{2}, \mathrm{CH}_{4}$ et $\mathrm{N}_{2} \mathrm{O}$;

- l'acidification, qui intègre les rejets de $\mathrm{NH}_{3}, \mathrm{NO}_{2}, \mathrm{NO}_{\mathrm{x}}$ et $\mathrm{SO}_{2}$;

- la toxicité terrestre, qui prend en compte les rejets de cadmium, cuivre, nickel, plomb et zinc ;

- l'utilisation d'énergie non renouvelable ;

- l'utilisation de pesticides ;

- 1'utilisation des surfaces agricoles.

Afin de pouvoir comparer les importances respectives de différentes catégories d'impacts, les effets ont été normalisés en les exprimant en pourcentage de l'impact global de l'ensemble des activités humaines. Pour ce faire, les impacts ont été calculés pour $54,4 \mathrm{~kg}$ de porc produit (consommation moyenne de porc en Europe de l'Ouest, OFIVAL 2002) et comparés aux impacts globaux des activités humaines par habitant (Huijbregts et al 2001). On voit alors que les quatre impacts environnementaux majeurs sont l'utilisation de surface, l'eutrophisation, l'acidification et le changement climatique (figure 17). Les impacts environnementaux des trois scénarios considérés sont présentés à la figure 18 , exprimés soit par kg de porc produit, soit par hectare de surface utilisée.

Figure 18. Impacts environnementaux de trois scénarios contrastés de production porcine (BPA : Bonne Pratiques Agricoles ; LR : Label Rouge ; $A B$ : Agriculture Biologique ; cf. tableau 4), mesurés par analyse du cycle de vie. Pour faciliter les comparaisons, chaque variable est transformée pour être comprise entre 0 et 1 (d'après Basset-Mens et Van der Werf 2005).

\section{Par kg de porc produit}

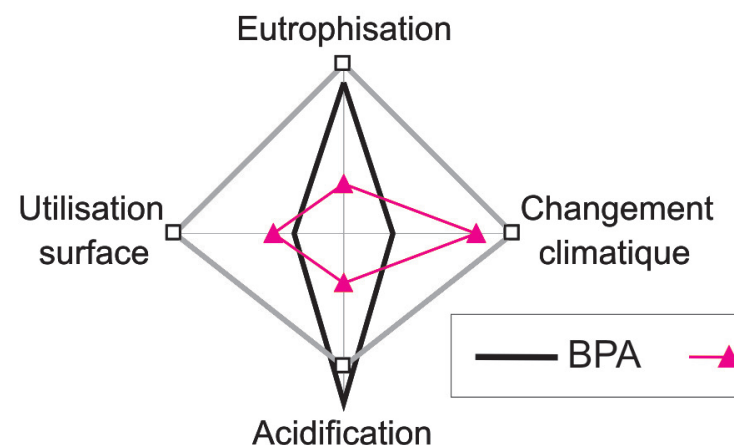

Acidification

\section{Par ha de surface utilisée}

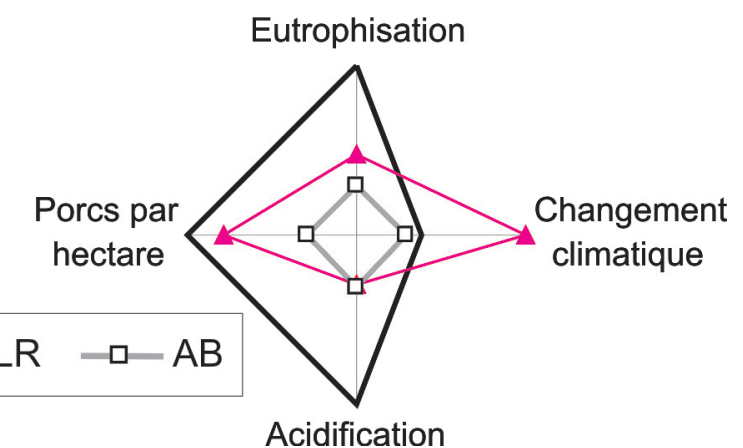


Figure 19. Contribution des différentes étapes de la production du porc aux impacts environnementaux, dans le cadre de trois scénarios contrastés (cf. tableau 4). Les valeurs sont exprimées en pourcentage de l'impact observé pour le scénario BPA. Les barres verticales indiquent les variations possibles, d'une variante basse à une variante haute, autour de la moyenne de l'impact total pour la variante de référence (d'après Basset-Mens et Van der Werf 2005).

a. Impact par kg de porc produit.

b. Impact par hectare.
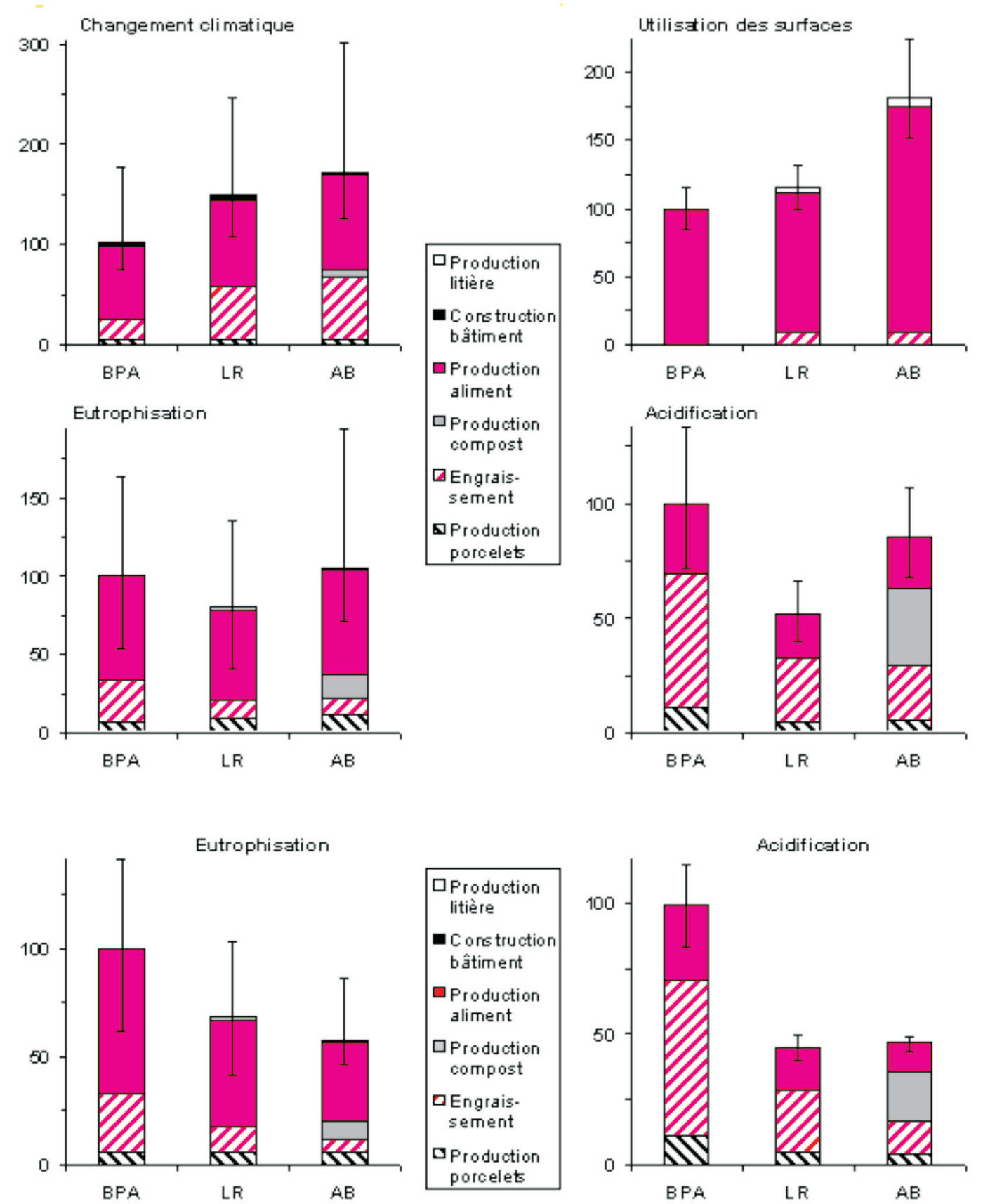

a) La production la plus intensive, suivant le scénario des bonnes pratiques agricoles, a le plus faible impact sur le changement climatique

S'agissant d'un impact global à l'échelle de la planète, il est préférable d'analyser l'impact sur le changement climatique en utilisant le mode d'expression par $\mathrm{kg}$ de porc produit. Le mode de production BPA contribue moins au changement climatique que les deux autres (figure 18). Les gaz à effet de serre sont émis pour l'essentiel au cours de la production d'aliments $\left(\mathrm{CO}_{2}\right.$ et $\mathrm{N}_{2} \mathrm{O}$ pour les cultures, $\mathrm{CO}_{2}$ pour les transports et la fabrication) et pendant la phase d'engraissement des animaux (figure 19a, cf. article sur les émissions gazeuses). Les différences d'impact entre scénarios proviennent des différences d'efficacité de la production (quantité de produit par unité d'intrant), aussi bien au niveau des cultures (production d'aliment) que de l'élevage des animaux (engraissement). Elles proviennent aussi du fait que l'élevage sur litière entraîne l'émission de gaz à effet de serre en quantité plus importante que l'élevage sur caillebotis (cf. article sur les émissions gazeuses). La variabilité/incertitude intra-scénario est très élevée (figure 19a), au moins aussi importante que les différences entre scénario, ce qui suggère qu'il reste dans toutes 
Figure 20. Origines principales des incertitudes sur les impacts environnementaux dans le cadre de trois scénarios contrastés de production porcine (cf. tableau 4). Pour chaque scénario trois variantes sont considérées : autour de la variante de référence, les variantes haute et basse permettent d'avoir une idée de la variabilité et des incertitudes intra-scénario. Les valeurs sont ramenées au $\mathrm{kg}$ de porc produit et exprimées en pourcentage de différence par rapport à l'impact observé pour la variante de référence du scénario BPA (d'après Basset-Mens et Van der Werf 2005).
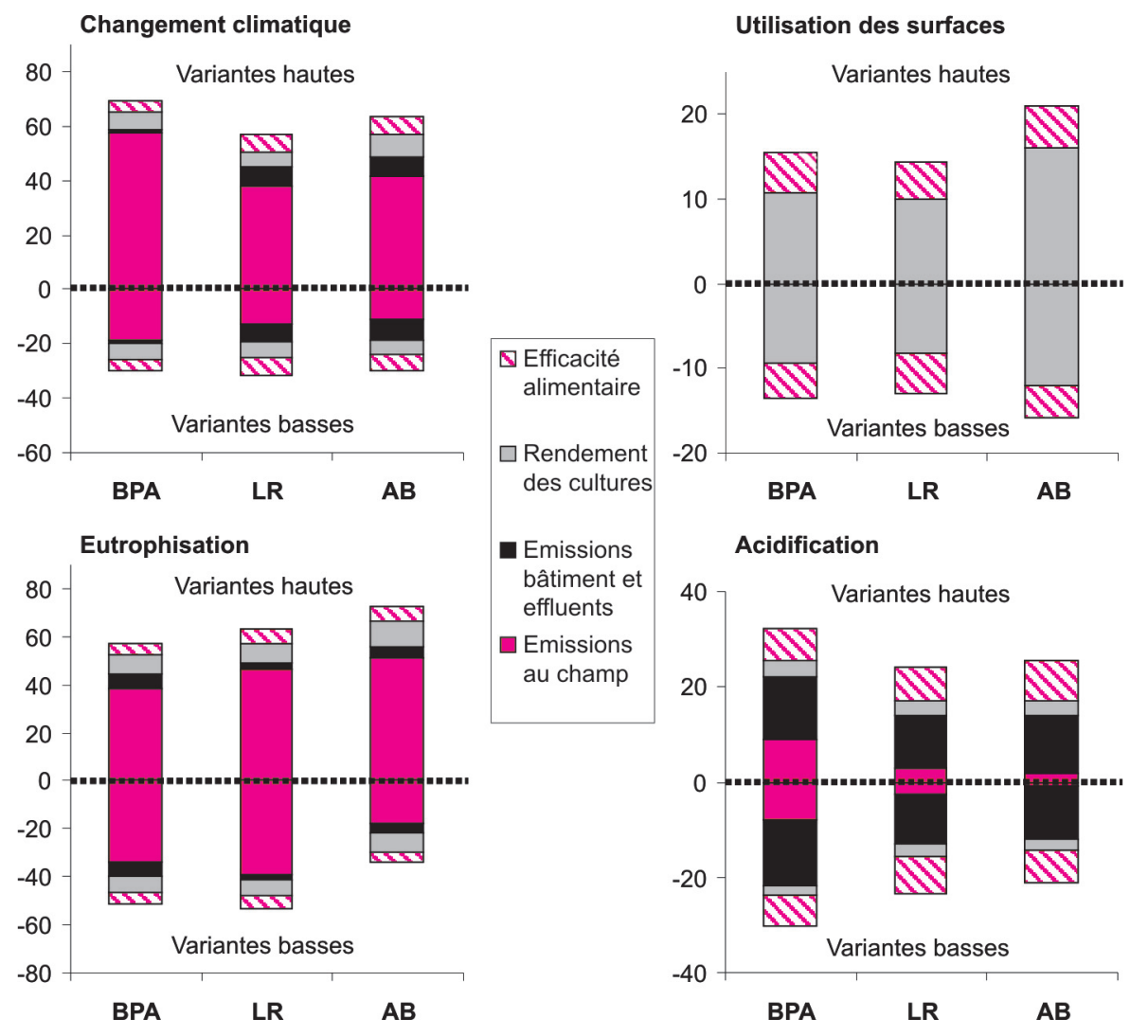

les situations de grandes marges de progrès au sein de chaque scénarios. La variabilité intra-scénario résulte pour l'essentiel des incertitudes sur les émissions au champ (figure 20) car les émissions de $\mathrm{N}_{2} \mathrm{O}$ dans le sol sont extrêmement difficiles à caractériser (cf. article sur les émissions gazeuses). La variabilité des émissions associée aux variations d'efficacité des activités d'élevage ou de production végétale est comparativement faible.

b) La production biologique, peu intensive, consomme beaucoup de surfaces

L'utilisation des surfaces doit s'analyser par kg de porc produit car il s'agit d'un impact global à l'échelle de la planète. Elle est en proportion directe du degré d'intensification des cultures qui fournissent l'aliment des animaux. Elle est logiquement beaucoup plus élevée pour l'agriculture biologique que pour les deux autres scénarios (figure 18) car les rendements sont moins élevés. Les marges de progrès les plus importantes

sont à trouver dans le rendement des cultures (figure 20) et, de façon moins marquée, dans l'efficacité alimentaire des animaux.

c) La production biologique a le plus faible impact sur l'eutrophisation

L'impact «eutrophisation» a des conséquences locales et il est donc préférable de l'analyser par ha de surface utilisée. Il est le plus faible pour le scénario $\mathrm{AB}$ (figure 18 ), en raison du caractère moins intensif de ce scénario au niveau des cultures produisant les aliments pour les animaux (figure 19b). Les émissions liées au compostage compensent le plus faible impact pendant l'engraissement en bâtiment. La variabilité/incertitude intra-scénario est très élevée (figure 19b). Elle résulte essentiellement des incertitudes sur les émissions au champ (figure 20) alors que la variabilité des émissions associées aux variations d'efficacité des activités d'élevage ou de production végétale est comparativement faible. d) Le mode de production selon les bonnes pratiques agricoles a le plus fort impact «acidification» ; la fabrication du compost accroit sensiblement les émissions acidifiantes dans le mode de production biologique

Les effets de l'acidification ont une portée à échelle continentale, intermédiaire entre les échelles mondiale et locale. Ils méritent donc d'être analysés selon les deux modes d'expression, par $\mathrm{kg}$ de porc produit et par hectare de surface utilisée.

Le mode de production BPA présente un effet d'acidification plus élevé que les deux autres, tout particulièrement lorsque l'impact est exprimé par hectare (figure 18). Les gaz acidifiants sont émis majoritairement en bâtiment pendant les phases d'élevage (production de porcelets, engraissement, figure 19). Dans le cas du scénario AB, les émissions de $\mathrm{NH}_{3}$ liées à la fabrication du compost (cf. article sur les émissions gazeuses) accroissent sensiblement l'impact acidification comparativement au scénario LR, l'amenant à un niveau proche de celui du scénario BPA. La variabilité/incertitude intra-scénario est modérée, comparativement à celle observée pour l'impact «changement climatique» (figure 19). Elle tient pour beaucoup à la variabilité des émissions dans le bâtiment et à partir des effluents (figure 20) ; c'est sans doute là que résident les plus grandes marges de progrès.
Ainsi, la hiérarchie des trois modes de production est très variable selon le type d'impact considéré, le scénario label rouge se trouvant en général en position intermédiaire entre les deux autres. Le scénario BPA a l'impact le plus élevé sur l'eutrophisation et l'acidification, mais il a l'impact le plus faible sur l'utilisation des surfaces et le changement climatique. Le mode de production biologique est sensiblement plus défavorable que les deux autres pour ce qui concerne les impacts globaux, à l'échelle de la planète (changement climatique et, surtout, utilisation des surfaces, figure 18). Il permet par contre de limiter les impacts locaux, du fait de son caractère moins intensif. A l'inverse, le mode de production le plus intensif (BPA) est très impactant à l'échelle locale mais se situe mieux que les deux autres pour le changement climatique du fait qu'il est très efficace en matière de transformation de l'énergie et des protéines, mais il est très défavorable en termes d'acidification. Il reste de fortes marges de progrès à exploiter au sein de chaque scénario. 
Tableau 6. Comment les trois études considérées dans cet article (1: Comparaison multicritères de deux types de conduite d'élevage; 2 : Optimisation au niveau de l'exploitation ; 3 : Evaluation globale de l'impact environnemental des élevages porcins) couvrent les différentes dimensions et les différentes échelles d'approche de la durabilité des systèmes de production

\begin{tabular}{|c|c|c|c|c|c|}
\hline \multirow[b]{2}{*}{ Dimensions des demandes } & \multicolumn{5}{|c|}{ Echelles d'approche } \\
\hline & Atelier $^{c}$ & Exploitation & Territoire $^{d}$ & Monde & Chaîne $^{e}$ \\
\hline \multicolumn{6}{|l|}{ Demandes sociales } \\
\hline Environnement & \multirow[b]{2}{*}{1} & 2 & & & \\
\hline $\begin{array}{l}\text { Nuisances } \\
\text { Bien-être animal }\end{array}$ & & & & & \\
\hline \multicolumn{6}{|l|}{$\begin{array}{l}\text { Demande des acteurs de la chaîne } \\
\text { de production porcine }\end{array}$} \\
\hline \multicolumn{6}{|l|}{ Travail } \\
\hline \multicolumn{6}{|l|}{ Rémunération } \\
\hline $\begin{array}{l}\text { - coûts de production } \\
\text { - valorisation/qualité produits }\end{array}$ & 1 & 2 & & & \\
\hline $\begin{array}{l}\text { Demande des consommateurs } \\
\text { Sécurité alimentaire }\end{array}$ & & & & & \\
\hline
\end{tabular}

\begin{abstract}
a Pour les besoins de ce tableau, les demandes qualitatives des consommateurs sont prises en compte dans les demandes des acteurs de la chaîne en matière de qualité des produits et dans les demandes sociales en matière de qualité des modes de production (environnement, bien-être).

b Assurer la quantité de nourriture suffisante pour nourrir la population. A ne pas confondre avec la sécurité sanitaire des aliments.

c Le terme «Atelier» désigne ici les bâtiments d'élevage et, le cas échéant, les surfaces extérieures occupées par les animaux.

d Le terme "Territoire» regroupe ici des entités géographiques de dimensions variables, depuis le voisinage immédiat de l'exploitation jusqu'à une région de programme. II peut aussi s'agir de l'aire d'action d'une entité économique.

e Chaîne de production depuis la fourniture des matières premières et des services pour l'élevage à la distribution des produits. Le terme "Chaîne» a été préféré dans cet article au terme «Filière», souvent employé, pour éviter toute confusion avec les filières de traitement des effluents évoquées au $\S 2$.
\end{abstract}

\section{Conclusions}

Les trois études considérées dans cet article couvrent de façons très différentes les différentes dimensions et échelles d'approche de la durabilité des systèmes de production (tableau 6).

L'étude expérimentale du paragraphe 1 sur la comparaison multicritères de deux types de conduite d'élevage aborde certes la plupart des dimensions pertinentes (sauf les aspects relatifs au travail) mais à une échelle réduite à celle de l'atelier porcin. Une optimisation du mode de production à cette seule échelle conduirait à des transferts de pollution et de problèmes vers l'extérieur de l'atelier, quitte à créer des incohérences au sein de l'exploitation, au sein de la chaîne de production, ou aux diverses échelles territoriales. Cette approche a cependant le mérite de faire s'affronter les différentes dimensions de la durabilité (environnementale, mais aussi sociale et économique), sur un même objet. Elle permet aussi de documenter les impacts environnementaux en les mesurant et non pas seulement en les estimant à l'aide de références plus ou moins arbitraires. C'est ainsi que les résultats obtenus par ce type d'approche expérimentale sont nécessaires pour alimenter les modèles construits pour couvrir des échelles plus vastes, sous réserve qu'ils aient un minimum de généricité, c'est-à-dire qu'ils ne soient pas totalement spécifiques des situations décrites dans l'expérience.

La modélisation mise en œuvre dans l'étude du paragraphe 2 aborde les interactions entre productions animales et végétales à l'échelle de l'exploitation, échelle très pertinente car c'est celle du centre décisionnel de base. Dans son état actuel de développement il s'agit encore d'une ébauche préliminaire qui demande à être améliorée et étendue. Le modèle reste très incomplet du fait qu'il ignore les dimensions non environnementales de la demande sociale, les aspects quantité et qualité du travail de l'exploitant ou de ses salariés. Par ailleurs, l'approche économique est limitée aux coûts de production, sans explorer les opportunités offertes par une possible meilleure valorisation des produits. L'analyse de l'impact environnemental est encore fruste, se limitant aux bilans $\mathrm{N}$ et $\mathrm{P}$ et aux émissions de $\mathrm{NH}_{3}$ et de $\mathrm{N}_{2} \mathrm{O}$. Enfin, du fait même que le modèle a fixé ses frontières au périmètre de l'exploitation, les risques de transfert de pollution et de problèmes hors des limites de l'exploitation sont bien réels et peuvent conduire à des incohérences aux échelles plus vastes. Toutefois, dans le principe, rien n'empêcherait de considérer dans ce type de modèle les impacts associés aux intrants de la même manière qu'ils sont considérés dans les approches ACV. Un des intérêts majeurs de la modélisation à l'échelle de l'exploitation est de révéler les interactions (positives ou négatives) entre gestion de l'environnement et rémunération de l'agriculteur. On pourrait utiliser ce type de modèle pour montrer comment il faudrait changer les modes de rémunération pour que les décisions de l'agriculteur aboutissent à une meilleure protection de l'environnement.

L'étude présentée au paragraphe 3 a conduit à choisir l'analyse du cycle de vie comme méthode d'évaluation de l'impact environnemental de l'élevage porcin. Les intérêts majeurs de cette méthode sont d'identifier d'éventuels transferts de pollution d'un élément de la chaîne à un autre et d'intégrer l'ensemble des effets de l'élevage dans un nombre restreint d'impacts environnementaux globalisés. En ce sens, elle répond bien à certaines des insuffisances des deux autres études. Néanmoins, dans son état actuel de développement, elle repose sur des valeurs d'émission standards trop imprécises pour bien décrire les situations particulières. L'intégration de modèles fonctionnant à des échelles plus restreintes en amont de l'ACV permettrait d'avoir des valeurs d'émissions plus réalistes et plus adaptées aux situations réelles étudiées. Par ailleurs, une limite majeure 
de l'approche dans son état de développement actuel est de se restreindre à la dimension environnementale.

Il n'est sans doute pas réaliste d'ambitionner la mise au point d'un super modèle intégrant l'ensemble des dimensions pertinentes à toutes les échelles. En fonction du problème à traiter, il convient de délimiter le périmètre le plus pertinent au sein de la matrice Dimensions x Echelles présentée au tableau 6. Pour gagner en précision, il faut s'efforcer d'utiliser les résultats obtenus aux échelles infra comme entrées pour les modèles développés aux échelles supérieures.

De façon générale, les meilleurs compromis entre coûts de production et respect de l'environnement sont obtenus par les systèmes les plus économes en surface (donc plus intensifs) qui utilisent au mieux les capacités du sol à produire de l'aliment pour les animaux et à recevoir leurs effluents (évitant ainsi le recours à des dépenses énergétiques pour le transport de matières premières et/ou le traitement des effluents). L'absence de lien au sol coûte cher en argent, mais aussi (surtout ?) en impact écologique. Un renforcement de ce lien au sol passe par une nouvelle façon de concevoir la répartition spatiale des élevages et les liens entre productions animales et végétales. Cette réflexion est à conduire au niveau de l'exploitation mais aussi de façon collective à des échelles plus larges.

\section{Références}

Basset-Mens C., van der Werf H., 2004 Evaluation environnementale de systèmes de production de porc contrastés. Journ. Rech. Porcine Fr., 36, 47-52.

Basset-Mens C., van der Werf H., 2005. Scénario-based environmental assessment of farming systems: the case of pig production in France. Agric. Ecosyst. Env., 105, 127-144

Baudon E., Cottais L., Leterme P., Espagnol S., Dourmad J.Y., 2005. Optimisation environnementale des systèmes de production porcine. Journ. Rech. Porcine Fr., 37, 325-332.

Béline F., Daumer M.L., Guiziou F., 2003. Performances des principales filières de traitement biologique aérobie du lisier de porcs. Journ. Rech. Porcine Fr., 35, 29-34

Carpentier A., Latouche K., Rainelli P., 2003. Les attributs de la viande de porc et la demande des consommateurs français. Journ. Rech. Porcine Fr., 35, 229-234.

COMIFER, 1996. Calcul de la fertilisation azotée des cultures annuelles. Guide méthodologique pour l'établissement des prescriptions locales. COMIFER, 85p.

COMIFER, 1997. Eléments complémentaires à la méthode de raisonnement de la fertilisation PK permettant d'aider à sa mise en œuvre. COMIFER, 28p.

CORPEN, 2003. Estimation des rejets d'azote, phosphore, potassium, cuivre et zinc des porcs. MAAPAR-MEDD, $44 \mathrm{p}$

Dourmad J.Y., Pomar C., Masse D., 2002. Modélisation du flux de composés à risque pour l'environnement dans un élevage porcin. Journ. Rech. Porcine Fr., 34, 183-194.
Dourmad J.Y., Baudon E., Espagnol S., Leterme P., 2005. Environmental optimization of swine production systems. Int. Workshop on green pork production, May 25-27, Paris, France, 169-170.

Dourmad J.Y., Leterme P., Salaün Y., Espagnol S., 2006. Intégration des différentes dimensions du fonctionnement d'une exploitation porcine au sein d'un simulateur. Rapport de synthèse de l'action AC54c du programme «Porcherie verte», 19p.

Dourmad J.Y., Hassouna M., Robin P., Guingand N., Meunier-Salaün M.C., Lebret B. 2008. Influence of pig rearing system on animal performance, manure composition and gaseous emissions. Animal (soumis).

Guinée J.B., Gorrée M., Heijungs R., Huppes G., Kleijn R., de Koning A., van Oers L., Wegener Sleeswijk A., Suh S., Udo de Haes H.A., de Bruijn H., van Duin R., Huijbregts M.A.J., 2002. Life Cycle Assessment. An operational guide to the ISO standards. Centre of Environmental Science, Leiden University, Leiden, The Netherlands.

Huijbregts M., Huppes G., de Koning A., van Oers L., Suh S., 2001. LCA normalisation factors for the Netherlands, Europe and the World. RIZA werkdocument 2001.059x, Leiden, The Netherlands.

Lebret B., Couvreur S., Meunier-Salaün M., Guigand N., Robin P., Hassouna M., Cariolet R., Dourmad J.Y., 2004. Comparaison expérimentale de deux conduites d'élevage de porcs en croissance. Journ. Rech. Porcine Fr., 36, 53-62.

Lebret B., Meunier-Salaün M., Bonhomme N., Foury A., Mormède P., Cariolet R., Guigand N., Robin P., Hassouna M., Le Floc'h N., Dransfield E., Dourmad J.Y., 2005. Comparaison expérimentale de deux conduites d'élevage de porcs en croissance. Rapport de synthèse de l'action AC54a du programme «Porcherie verte», $35 \mathrm{p}$.

Lebret B., Meunier-Salaün M.C., Foury A., Mormède P., Dransfield E., Dourmad J.Y., 2006a. Influence of rearing conditions on performance, behavioral and physiological responses of pigs to preslaughter handling, carcass traits and meat quality. J. Anim. Sci., 84 2436-2447.

Lebret B., Meunier-Salaün M.C., Foury A., Mormède P., Dransfield E., Dourmad J.Y. 2006b. Effets du mode d'élevage sur les performances, le comportement des animaux et la qualité de la viande chez deux génotypes de porcs. Journ. Rech. Porcine Fr., 38, 81-88.

Levasseur P., le Bris B., Gorius H., Le Cozler Y., 2003. Traitement biologique par boue activée et compostage du lisier sur paille : enquête en élevage. Techni-Porc, 26, 5-11.

Meunier-Salaün M.C., Dourmad J.Y., Lebret B., 2006. Evaluation comparée de deux systèmes d'élevage par la réponse comportementale des porcs à l'introduction d'un nouvel objet dans le milieu de vie. Journ. Rech. Porcine Fr., 38, 417-422.

OFIVAL, 2002. Chiffres clés 2001. FranceUnion européenne-Monde. Les cahiers de l'OFIVAL, Paris, France.

Van der Werf H.M.G., Petit J., 2002. Evaluation of the environmental impact of agriculture at the farm level: a comparison and analysis of 12 indicator-based methods. Agric. Ecosyst. Env., 93, 131-145.

Van der Werf H.M.G., Tzilivakis J., Lewis K., Basset-Mens C., 2007. Environmental impacts of farm scenarios according to five assessment methods. Agric. Ecosyst. Env., 118, 327-338.

\section{Résumé}

Cet article résume les apports du programme «Porcherie verte» dans l'évaluation globale intégrée des systèmes de production porcine, prenant en compte à différentes échelles les diverses dimensions pertinentes de la durabilité, au-delà des seuls impacts environnementaux. La comparaison multicritères de deux types de conduite d'élevage prend en compte l'ensemble des dimensions de la durabilité (environnement, société, économie) mais à une échelle restreinte à l'atelier d'élevage porcin. Comparativement aux animaux élevés sur caillebotis, les porcs élevés sur litière avec accès à une courette bénéficient d'un meilleur bien-être, ont de meilleures performances de croissance mais leurs carcasses peuvent être plus grasses. Leurs viandes peuvent avoir une moindre qualité technologique, mais une meilleure qualité sensorielle. Ce type de conduite produit nettement moins d'odeurs désagréables mais il est plus coûteux. Les produits peuvent cependant potentiellement être mieux valorisés par un label. 
La modélisation visant une optimisation au niveau de l'exploitation prend en compte les dimensions environnementales et économiques, mais pas sociétales, pour comparer différentes filières de gestion des effluents. L'échelle d'approche est plus large que dans l'étude précédente pour prendre en compte les interactions entre productions animales et végétales sur le territoire de l'exploitation qui correspond à un échelon décisionnel fondamental en agriculture. La filière «Lisier brut» conduit à la meilleure marge brute marginale, avec de bons bilans environnementaux, mais la production est limitée à 60 porcs à l'ha. Le traitement biologique du lisier sans séparation de phases permet d'augmenter la production de porcs mais détériore le bilan apparent de phosphore de l'exploitation et réduit significativement la marge brute par porc produit. La mise en ouvre d'un procédé de séparation de phases préalablement au traitement biologique du lisier permet d'augmenter encore la production de porcs en respectant des contraintes environnementales sur le phosphore, mais il réduit encore plus la marge brute par porc produit. Le compostage du lisier peut être une alternative au traitement biologique pour les petites exploitations, mais son bilan environnemental est médiocre. L'exportation du compost de lisier allège les bilans apparents de $\mathbf{N}$ et de $\mathbf{P}$ de l'exploitation mais ne change pas les émissions gazeuses. Il permet de maintenir une bonne marge brute, même aux chargements élevés. L'élevage de porcs sur litière permet de produire plus de porcs à l'ha mais au prix d'une dégradation de l'impact environnemental et d'une augmentation des coûts de production. Le compostage du fumier des litières permet d'augmenter la production de porcs à l'ha, mais uniquement en l'absence de contrainte environnementale sur le phosphore, et l'accroissement marginal de marge brute est faible. L'exportation du compost excédentaire permet d'augmenter encore la production de porcs en allégeant les bilans apparents de $\mathbf{N}$ et $\mathbf{P}$, mais pas les émissions gazeuses. Parmi les filières avec traitement, qui autorisent des chargements élevés, le traitement biologique semble réaliser le meilleur compromis entre marge brute réalisée et impact sur l'environnement, si l'on néglige les transferts d'impacts hors de l'exploitation. Parmi les filières avec traitement et exportation, qui permettent des niveaux de chargement très élevés, le compostage de fumier semble cumuler les inconvénients en termes de marge brute et d'impacts environnementaux, si l'on néglige les transferts d'impacts hors de l'exploitation associés au traitement biologique.

L'évaluation globale de l'impact environnemental des élevages porcins est restreinte à la dimension environnementale mais son échelle d'approche permet d'intégrer l'ensemble des impacts associés aux processus en amont de l'exploitation L'analyse de cycle de vie (ACV) est une méthode de choix pour faire cette évaluation et a été mise en ouvre pour comparer les impacts environnementaux de trois scénarios contrastés de production porcine. La production la plus intensive, suivant le scénario des bonnes pratiques agricoles, a le plus faible impact sur le changement climatique, mais le plus fort impact sur l'acidification. La production biologique, peu intensive, consomme beaucoup de surfaces, mais elle a le plus faible impact sur l'eutrophisation.

De façon générale, les meilleurs compromis entre coûts de production et respect de l'environnement sont obtenus par les systèmes les plus économes en surface, qui utilisent au mieux les capacités du sol à produire de l'aliment pour les animaux et à recevoir leurs effluents. L'absence de lien au sol coûte cher en argent et en impact écologique.

\section{Abstract}

\section{Overall evaluation of pig production systems and their optimisation on the farm level}

This paper summarises the contributions made by the «Porcherie verte» programme in the overall integrated evaluation of pig production systems, considering on different scales the different pertinent aspects of sustainability, beyond environmental impact. The multiple criteria comparison of two types of breeding management considers all the aspects of sustainability (environment, society, economy) but at a level restrained to pig breeding. Pigs raised on litter with access to a small courtyard have better welfare, better growth performances but with fatter carcasses than pigs raised on wood slats. Their meat has a poorer technological quality but a better sensorial quality. This type of management produces less unpleasant odours but is more costly. The products can, however, be better valorised by a label.

Modelling aimed at optimisation of a farm considers the environmental and economic aspects, but not those of society, when comparing the different ways to manage waste. The scale of the approach is larger than in the preceding study, considering the interactions between animal and plant production on the farm's property, which corresponds to a fundamental decision scale in agriculture. The «raw manure» waste management leads to an improved gross margin, with good environmental balance, but the production is limited to 60 pigs per hectare. The biological treatment of manure without phase separation allows increasing pig production but deteriorates the phosphorus balance of the farm and significantly reduces the gross margin per pig produced. Phase separation before biological treatment of manure allows an increased pig production that respects environmental phosphorus constraints, but it decreases the gross margin per pig produced. Manure composting can be an alternative to biological treatment for small farms, but its environmental balance is poor. Exporting manure compost slightly improves the apparent $\mathbf{N}$ and $\mathbf{P}$ balances of the farms but does not change gas emissions. It allows maintaining good gross emissions, even with high numbers of animals. Pig breeding on litter allows the production of more pigs/ha but is unfavourable for the environmental impact and increases production costs. Composting litter manure increases pig production per ha but only in the absence of environmental constraints on phosphorus and the growth of the gross margin is low. Exporting the compost surplus allows increasing pig production by lowering the apparent $\mathbf{N}$ and $\mathbf{P}$ balances, but not gas emissions. Biological treatment seems to give the best compromise between gross margin and environmental impact amongst the different types of treatment that allow a large number of animals, as long as one does not consider the impact of transfers outside of the farm. Amongst the different types of treatment with exportation that allow very high population levels, the composting of manure seems to cumulate the disadvantages in terms of gross margin and environmental impacts, if one neglects the impact of transfers outside of the farm associated with biological treatment.

The global evaluation of environmental impact of pig farms is restrained to the environmental aspects but its scale of approach integrates all the impacts associated with the processes upstream of the farm. The analysis of the life cycle (ACV) is a method of choice to do this evaluation and was developed for the comparison of the environmental impacts of the three contrasted scenarios of pig production. The most intensive production according to the scenario of good agricultural practices has the lowest impact on climate change, but the highest impact on acidification. Biological production that is not intensive uses a large surface but has a lower impact on eutrophisation. 
Generally, the best compromise between production costs and environmental respect are obtained by the most economic systems in terms of surface, which uses the soil's capacities the best to produce feed for animals and receive their waste. The absence of a link to soil is expensive and has a high impact on ecology.

BONNEAU M., DOURMAD J.-Y., LEBRET B., MEUNIER-SALAÜN M.-C., ESPAGNOL S., SALAÜN Y., LETERME P., VAN DER WERF H., 2008. Evaluation globale des systèmes de production porcine et leur optimisation au niveau de l'exploitation. INRA Prod. Anim., 21, 367-386. 